\title{
Changes in Prefrontal Axons May Disrupt the Network in Autism
}

\author{
Basilis Zikopoulos ${ }^{1,2}$ and Helen Barbas ${ }^{1,2,3}$ \\ ${ }^{1}$ Neural Systems Laboratory and ${ }^{2}$ Program in Neuroscience, Boston University, Boston, Massachusetts 02215, and ${ }^{3}$ New England Regional Primate \\ Research Center, Harvard Medical School, Boston, Massachusetts 01772
}

Neural communication is disrupted in autism by unknown mechanisms. Here, we examined whether in autism there are changes in axons, which are the conduit for neural communication. We investigated single axons and their ultrastructure in the white matter of postmortem human brain tissue below the anterior cingulate cortex (ACC), orbitofrontal cortex (OFC), and lateral prefrontal cortex (LPFC), which are associated with attention, social interactions, and emotions, and have been consistently implicated in the pathology of autism. Area-specific changes below ACC (area 32) included a decrease in the largest axons that communicate over long distances. In addition, below ACC there was overexpression of the growth-associated protein $43 \mathrm{kDa}$ accompanied by excessive number of thin axons that link neighboring areas. In OFC (area 11), axons had decreased myelin thickness. Axon features below LPFC (area 46) appeared to be unaffected, but the altered white matter composition below ACC and OFC changed the relationships among all prefrontal areas examined, and could indirectly affect LPFC function. These findings provide a mechanism for disconnection of long-distance pathways, excessive connections between neighboring areas, and inefficiency in pathways for emotions, and may help explain why individuals with autism do not adequately shift attention, engage in repetitive behavior, and avoid social interactions. These changes below specific prefrontal areas appear to be linked through a cascade of developmental events affecting axon growth and guidance, and suggest targeting the associated signaling pathways for therapeutic interventions in autism.

\section{Introduction}

Communication problems are at the core of the entire spectrum of autism disorders, disrupting particularly the social interactions of affected individuals. Genetic studies in autism have implicated changes in expression of genes that affect connectivity and neuronal excitability (Morrow et al., 2008; Glessner et al., 2009; Weiss et al., 2009) (for review, see (Rubenstein and Merzenich, 2003; Walsh et al., 2008). At the brain level, studies have identified functional abnormalities in neural networks in autism that prominently involve the frontal cortex (Barnea-Goraly et al., 2004; Casanova, 2004; Herbert et al., 2004; Courchesne and Pierce, 2005; Just et al., 2007) and functionally related distant association areas (Just et al., 2007; Koshino et al., 2008). Interestingly, the white matter below the frontal lobe is enlarged in young children with autism but not in adults, as assessed by structural imaging (Herbert et al., 2004). However, adults with autism continue to exhibit deficits associated with the disorder, and imaging studies show decreased functional connectivity among brain ar-

\footnotetext{
Received April 30, 2010; revised July 26, 2010; accepted Aug. 29, 2010.

This work was supported by Autism Speaks, and National Institutes of Health grants from National Institute of Mental Health, National Institute of Neurological Disorders and Stroke, and National Science Foundation Center of Excellence for Learning in Education, Science, and Technology. We thank Clare Timbie, Amar Patel, Mary Louise Fowler, Seung Yeon Michelle Kim, and Sue Paul for technical assistance; and Marcia Feinberg for assistance with electron microscopy. We also thank Dr. Alan Peters and Dr. Claus Hilgetag for useful comments. We gratefully acknowledge the Autism Tissue Program and the Harvard Brain Tissue Resource Center for providing human brain tissue.

Correspondence should be addressed to Helen Barbas, Boston University, 635 Commonwealth Avenue, Room 431, Boston, MA 02215. E-mail: barbas@bu.edu.

DOI:10.1523/JNEUROSCI.2257-10.2010

Copyright $\odot 2010$ the authors $\quad$ 0270-6474/10/3014595-15\$15.00/0
}

eas, desynchronization of cortical activity, and changes in the fractional anisotropy of the white matter (Barnea-Goraly et al., 2004; Kana et al., 2006; Just et al., 2007; Keller et al., 2007; Minshew and Williams, 2007; Koshino et al., 2008; Thakkar et al., 2008; Minshew and Keller, 2010). These findings suggest compromise of the structural integrity of the white matter that may be below the resolution of magnetic resonance imaging.

Despite evidence indicating disruption of cortical pathways in autism, there is no information as to whether there are structural defects in single axons, which are the conduit for neural communication. To address this issue, we investigated the fine structure of myelinated axons in postmortem brain tissue of adults with autism and matched controls (Table 1 lists cases and clinical characteristics). We investigated exclusively myelinated axons because they make up the large majority ( $\sim 90 \%)$ of axons (LaMantia and Rakic, 1990a), and focused on the white matter below the following three prefrontal regions: anterior cingulate cortex (ACC); orbitofrontal cortex (OFC); and lateral prefrontal cortex (LPFC). These functionally distinct regions are associated with attention, emotions, and executive function in processes that are severely affected in autism (Luna et al., 2002; Courchesne and Pierce, 2005; Bachevalier and Loveland, 2006; Hardan et al., 2006; Girgis et al., 2007; Loveland et al., 2008; Thakkar et al., 2008; Griebling et al., 2010).

\section{Materials and Methods}

Experimental design. The objective was to investigate whether or not abnormalities of the white matter below frontal areas in autism observed with structural imaging in children persist in the brains of adults with 

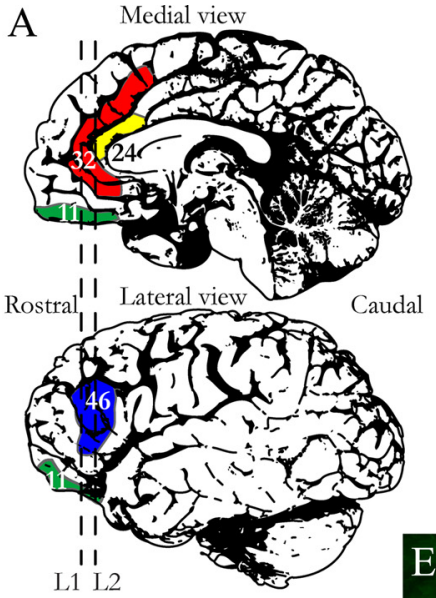

$\mathrm{D}$
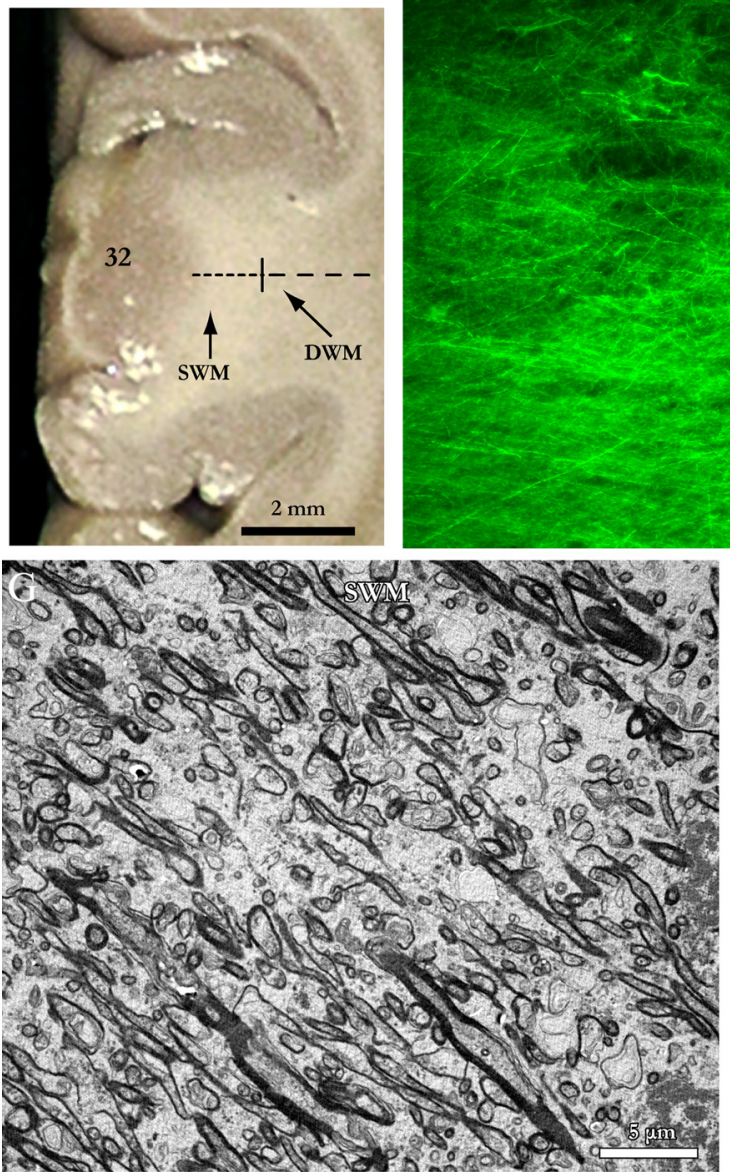

B

$\mathrm{E}$

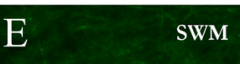

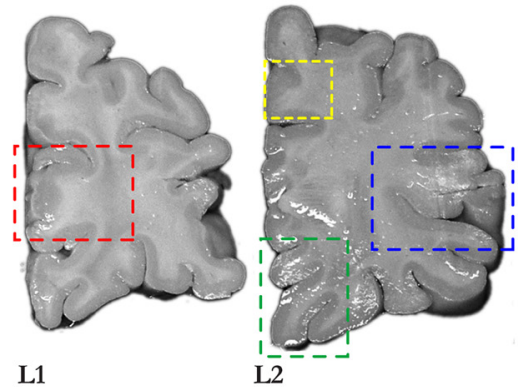
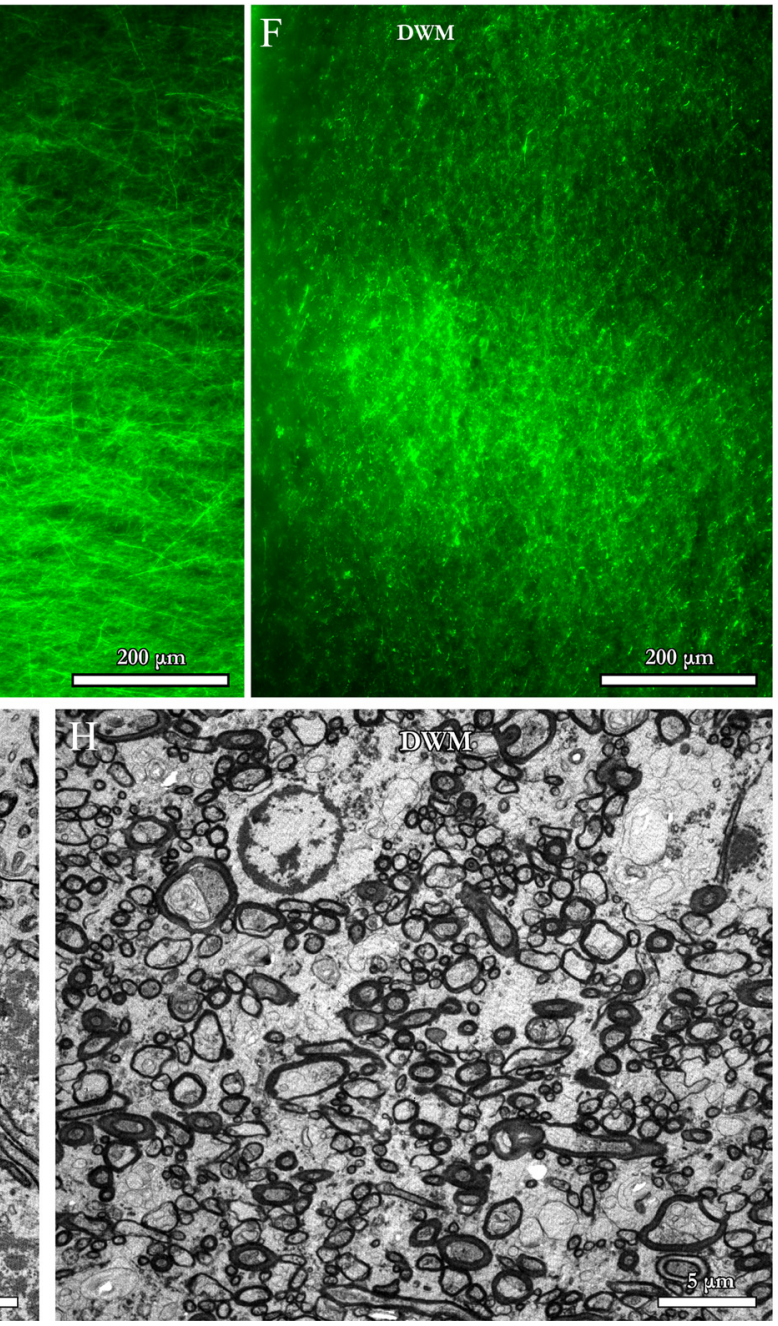

Figure 1. Map of prefrontal areas studied and segmentation of the white matter. $A$, Medial (top) and lateral (bottom) views of the human brain show the three prefrontal areas studied; $A C C$ ( $A 32$, red; anterior A24, yellow); OFC (A11, green); LPFC (A46, blue). Dotted lines indicate the levels (L1, L2) used for analysis. B, 1-cm-thick slabs of frontal cortex show the areas sampled (color-coded dotted-line squares: A32, red; A24, yellow; A11, green; A46, blue). C, Matched levels from the brain atlas from the Autism Tissue Portal. D, Coronal view of a representative ACC (A32) tissue slab. Dotted lines indicate gross (macroscopic) distinction of superficial (SWM) and deep (DWM) white matter, based on subsequent microscopic analysis. $\boldsymbol{E}$, $\boldsymbol{F}$, Fluorescent photomicrographs of coronal sections from A32 white matter after labeling of axons with NFP-200 (green). Light microscopic segmentation of superficial $(\boldsymbol{E})$ and deep $(\boldsymbol{F})$ white matter is based on the distinct orientation of axons at different depths from the gray matter. Axons in the superficial white matter travel mainly perpendicular to the surface of the cortex (E, axons appear mainly as thin lines), whereas in the deep white matter most axons travel parallel to the cortical surface ( $\boldsymbol{F}$, axons appear mainly as green dots). $\boldsymbol{G}, \boldsymbol{H}$, EM photomicrographs show the prevalence of elongated axon profiles in the superficial white matter $(\boldsymbol{G})$ and the prevalence of circular axon profiles in the deep white matter $(\boldsymbol{H})$.

autism. We used unbiased quantitative stereology to study myelinated axons at high resolution at the light microscope (LM) and their fine structure at the electron microscope (EM) below the ACC (A32), OFC (A11), and LPFC (A46) areas (Fig. $1 A-C)$ in the brains of autistic $(n=5$, 1 female) and age-matched, typically developed controls $(n=4,2 \mathrm{fe}-$ males). We investigated the density of axons and thickness of axons and myelin sheaths. We examined only myelinated axons because they constitute the vast majority of axons in the frontal cortical white matter $(\sim 90 \%)$, the corpus callosum, and anterior and hippocampal commissures in primates (LaMantia and Rakic, 1990a). Further, myelinated ax- 
Table 1. Clinical characteristics of postmortem cases and prefrontal areas studied

\begin{tabular}{|c|c|c|c|c|c|c|c|}
\hline Case number & Diagnosis & Age at death (years) & Sex & Postmortem interval (h) & Primary cause of death & Hemisphere & Areas used $^{a}$ \\
\hline B-4786 & Control & 36 & $M$ & 20 & Myocardial Infarction & Right & $11,24,32,46$ \\
\hline B-4981 & Control & 42 & $M$ & 18 & Myocardial Infarction & Right & $11,24,32,46$ \\
\hline B-5353 & Control & 41 & $\mathrm{~F}$ & 14 & Unknown & Right & $11,24,46$ \\
\hline B-6004 & Control & 36 & $\mathrm{~F}$ & 18 & Unknown & Right & $11,24,32,46$ \\
\hline $\mathrm{B}-4541^{b}$ & Autism & 44 & M & 31 & Acute Myocardial Infarction & Right & $11,24,32,46$ \\
\hline B- $4871^{C}$ & Autism & 31 & $M$ & 99 & Shooting & Right & $11,24,32,46$ \\
\hline$B-5173^{d}$ & Autism & 30 & M & 20 & Gastro-Intestinal Bleeding & Right & $11,24,32,46$ \\
\hline B-6232 & Autism & 40 & $\mathrm{~F}$ & 33 & Respiratory Arrest & Left & $11,24,32,46$ \\
\hline B-6677 & Autism & 30 & $M$ & 16 & Congestive heart failure & Right & $11,24,32,46$ \\
\hline
\end{tabular}

$M$, male; F, female.

${ }^{a}$ Areas examined depended on tissue availability in matched subjects. Area 24 was used only for the examination of GAP-43 levels in autistic and control cases.

Other diagnosed disorders included ${ }^{b}$ schizophrenia, ${ }^{c}$ depression, and ${ }^{d}$ seizures.

ons can be labeled using immunohistochemical methods, which we used for an independent evaluation at the light microscope.

White matter segmentation. We investigated axons in the superficial and deep white matter separately for two reasons. First, structural imaging studies suggested possible differences in pathology in autism (Herbert et al., 2004). And second, the deep white matter contains axons that communicate over long distances, whereas the superficial white matter contains axons that communicate mostly over short or medium distances (Schmahmann and Pandya, 2006). We thus divided the white matter into superficial (outer or radiate) and deep (inner or sagittal) compartments, based on axon orientation and distance from the cortical gray matter (Meyer et al., 1999). We determined axon alignment at the LM and EM in serial coronal ultrathin sections at gradually increasing distances from the gray matter-white matter border. The superficial compartment included axons that were mostly aligned radially and were immediately adjacent to layer VI of the overlying cortical areas (at a distance up to 2 $\mathrm{mm}$ from layer VI). The deep compartment included axons that run mainly sagittally and more or less parallel to the cerebral surface (Fig. $1 D-H)$.

Tissue preparation. Postmortem prefrontal brain tissue was obtained from the Harvard Brain Tissue Resource Center through the Autism Tissue Program from five autistic adults (one female) and four typically developed, age-matched controls (two females). The selection of matched cases used was based on tissue availability. The study was approved by the Institutional Review Board of Boston University. The diagnosis of autism was based on the Autism Diagnostic Interview-Revised in all cases (supplemental Table 1, available at www.jneurosci.org as supplemental material). Clinical characteristics are summarized in Table 1 and supplemental Table 1 (available at www.jneurosci.org as supplemental material). Some autistic cases were diagnosed with seizure disorder (case 5173), depression (case 4871), and schizophrenia (case 4541). Results from the analysis of the features of axons in these and the three female cases did not differ from other cases within each group in this and other studies that used tissue from the same cases (Buxhoeveden et al., 2006; Schumann and Amaral, 2006; Yip et al., 2007).

We excised small blocks $(\sim 2 \times 3 \mathrm{~cm})$ of matched ACC (A32, A24), OFC (A11), and LPFC (A46) cortices containing gray and white matter (Fig. $1 A-C$ ) based on the human brain atlas from the Autism Tissue Portal (www.atpportal.org) and (reissued, von Economo, 2009) and additional cytoarchitectonic studies of human prefrontal cortex (Selemon et al., 1998; Stark et al., 2004; Miguel-Hidalgo et al., 2006).

We postfixed tissue blocks in 2\% paraformaldehyde and 2.5\% glutaraldehyde, in $0.1 \mathrm{~m}$ phosphate buffer (PB), $\mathrm{pH} 7.4$, for $2 \mathrm{~d}$ at $4^{\circ} \mathrm{C}$. To preserve the ultrastructure until processing, tissue blocks were immersed in antifreeze solution (30\% ethylene glycol, $30 \%$ glycerol, $40 \% 0.05 \mathrm{M} \mathrm{PB}$, $\mathrm{pH} 7.4$, with $0.05 \%$ azide) and stored at $-20^{\circ} \mathrm{C}$. The blocks were then rinsed in $0.1 \mathrm{M} \mathrm{PB}$ and cut coronally in $50-\mu \mathrm{m}$-thick sections on a vibratome (series 1000, Pelco). In all cases, tissue blocks through the gray matter of the areas of the associated white matter sampled were frozen in $-70^{\circ} \mathrm{C}$ isopentane, cut in a cryostat (CM 1500, Leica) in the coronal plane at $20 \mu \mathrm{m}$ in 10 series, and mounted on chrome alum-coated slides.

Immunohistochemistry. We conducted several immunoassays to label specific axon features and white matter oligodendrocytes. At the light microscope, we labeled oligodendrocytes with an antibody against myelin- and oligodendrocyte-specific protein (MOSP). We also labeled myelinated axons with an antibody against neurofilament protein 200 $\mathrm{kDa}$ (NFP-200), and examined branching axons with an antibody for growth-associated protein $43 \mathrm{kDa}$ (GAP-43). To sort out axons from the thalamus, we used antibodies against calbindin (CB) and parvalbumin (PV), which label excitatory thalamic projections to the cortex, and examined labeling at the confocal microscope and EM.

Series of free-floating coronal tissue sections ( $50 \mu \mathrm{m}$ thick) or cryosections mounted on slides ( $20 \mu \mathrm{m}$ thick) were used in all immunohistochemical procedures. Sections were rinsed in $0.01 \mathrm{~m} \mathrm{PBS,} \mathrm{pH} \mathrm{7.4,}$ followed by $10 \%$ normal goat serum, $5 \%$ bovine serum albumin, and $0.1 \%$ Triton X-100 in $0.01 \mathrm{~m}$ PBS blocking solution for $1 \mathrm{~h}$ and incubated for $1-2 \mathrm{~d}$ in primary antibody.

We labeled axons in the white matter with antibodies against $\mathrm{CB}$ (mouse monoclonal; dilution 1:1000; Swant and/or Sigma), PV (rabbit polyclonal; dilution 1:1000; Swant and/or Sigma), NFP-200 (rabbit polyclonal; dilution 1:200; Millipore Bioscience Research Reagents), and GAP-43 (mouse monoclonal; dilution 1:2000; Millipore Bioscience Research Reagents). We labeled oligodendrocytes with a monoclonal antibody against MOSP (mouse monoclonal; dilution 1:1000; Millipore Bioscience Research Reagents). The sections were rinsed in PBS, incubated for $4 \mathrm{~h}$ with goat anti-mouse or anti-rabbit secondary antibodies conjugated with the fluorescent probes Alexa Fluor 488 (green) or 568 (red; 1:100; Invitrogen) and thoroughly rinsed with PBS. In some cases, a biotinylated secondary antibody and an avidin-biotin-peroxidase kit were used to label CB-positive or PV-positive axons with diaminobenzidine (Zymed Laboratories), which were further processed for EM (see Electron microscopy, below). To test for nonspecific labeling, we performed control experiments with sections adjacent to those used in the experiments. These included omission of the primary antibodies and incubation with secondary antisera. Control experiments showed no immunohistochemical labeling.

Electron microscopy. Tissue processing and pre-embedding immunohistochemical labeling for serial EM are especially challenging techniques for postmortem human brain tissue because of limited control over tissue extraction protocols, length of the postmortem interval, and use of fixation. In addition, processing and labeling of the tissue can degrade the ultrastructure and preclude quantitative analyses. To address these issues, we have developed several novel protocols that maximize tissue quality and specificity of labeling (Zikopoulos and Barbas, 2006, 2007). We preserve tissue blocks or sections at $-20^{\circ} \mathrm{C}$ in antifreeze buffer solution for long periods of time, fix and process tissue using a variable microwave, and label tissue before embedding, all of which enhance and accelerate penetration of reagents in brain sections during processing, reduce nonspecific background staining, minimize the need for detergents that degrade fine structure, and decrease potential damage of a series. These protocols have markedly increased tissue quality and made it possible to conduct three-dimensional (3D) quantitative reconstruction of identified structures.

Sections were rinsed briefly in $0.1 \mathrm{M} \mathrm{PB}$ and postfixed in a variable wattage microwave oven (Biowave, Pelco) with $6 \%$ glutaraldehyde at 150 W. Small blocks of sections containing the outer (superficial) or inner 
(deep) parts of the white matter below prefrontal cortices were cut under a dissecting microscope, postfixed in $1 \%$ osmium tetroxide with $1.5 \%$ potassium ferrocyanide in $\mathrm{PB}$, washed in buffer $(\mathrm{PB})$ and water, and dehydrated in an ascending series of alcohols. While in $70 \%$ alcohol, they were stained with $1 \%$ uranyl acetate for $30 \mathrm{~min}$. Tissue sections were then cleared in propylene oxide and embedded in Araldite at $60^{\circ} \mathrm{C}$. Serial ultrathin sections $(50 \mathrm{~nm})$ were cut in the horizontal plane with a diamond knife (Diatome) using an ultramicrotome (Ultracut, Leica) and collected on single-slot grids to view with a transmission electron microscope (100CX, Jeol), as previously described (Zikopoulos and Barbas, 2006, 2007). Myelinated axons were easily identified at the EM by the darkly stained electron dense myelin sheath (Peters et al., 1991).

Nissl staining. One series of sections was stained for Nissl using thionin to view neurons and glia and to examine the cytoarchitecture of each area, as previously described (Barbas and Pandya, 1989; Dombrowski et al., 2001). Sections were dried, defatted in a 1:1 solution of chloroform and $100 \%$ ethanol for $1 \mathrm{~h}$, rehydrated through a series of graded alcohols and $\mathrm{dH}_{2} \mathrm{O}$, stained with $0.05 \%$ thionin, $\mathrm{pH} 4.5$, for $15 \mathrm{~min}$, differentiated through graded alcohols and xylenes, and coverslipped with Entellan (Merck).

Sample size. To determine adequate sample size, we performed an $a$ priori power analysis, using repeated measures from a pilot study, and an a posteriori power analysis, using the actual data. The a posteriori power analysis took into consideration all known and estimated variables, including age, sex, postmortem interval (PMI), and other diagnoses, and was used to test the validity of the pilot study and the a priori power analysis, which always rely on fewer data points and make more assumptions. These analyses, which had an estimated large effect size in the population, 0.80 , showed that the sampling ratios that were used exceeded the samples needed to detect differences with a $>90 \%$ probability. We used several additional computational and statistical methods to establish adequate sample size, including progressive means analysis with exhaustive sampling, and the formula of West et al. (1991). We used higher sampling fractions in all analyses than the minimum of three cases and three sections required by the power analyses, and in most cases we expanded these numbers to five brains from autistic individuals, four brains from control cases, and more than five sections per case. In all analyses, the sample size included number of cases, volume fraction of areas sampled, and number of individual axons examined, which were not only adequate but exceeded the estimated minimum requirements. Moreover, for each case we examined thousands of axons at very high resolution, totaling nearly 50,000 for the study. In one analysis (A32), tissue was available only for three control cases ( 1 female and 2 male).

Stereological analysis at the LM. We estimated the overall and laminar density of neurons in A32, A11, and A46 overlying the sites of white matter analysis, and the density of oligodendroglial cells in the superficial part of the white matter below OFC (A11) using the unbiased stereological method of the optical fractionator (Gundersen, 1986; Howard and Reed, 1998) with the aid of commercial software (StereoInvestigator, Microbrightfield), as previously described (Zikopoulos and Barbas, 2006). For LM quantitative analyses, we used a minimum of three sections from one series of coronal sections (20 $\mu \mathrm{m}$ thick) from each case. To ensure an unbiased estimate of the number of neurons, we first measured the thickness of each section, and used StereoInvestigator to set a guard zone at the bottom and top of each section to correct for objects plucked during sectioning; the disector thickness was thus smaller than the thickness of the section (Gundersen, 1986; West et al., 1991; Howard and Reed, 1998). The sampling fraction was one-fiftieth of the total volume of the area examined, and was determined in pilot studies using exhaustive sampling and progressive means analysis so that final estimates had an $\mathrm{SE} \leq 10 \%$. The use of uniform random sampling ensured that every part of the area examined had the same chance of being included in the sample. The numbers of neurons and volumes of the corresponding area and layers estimated with the Cavalieri method were divided to assess the density of neurons in each case. We normalized data by expressing the density of neurons as a percentage of the total density of all labeled neurons in each area in each case.

For the analysis of the number of myelinated axons with branches, and to estimate the number of axons that express GAP-43, we double labeled a minimum of three sections from one series per case with NFP-200 and GAP-43 and used systematic random sampling (sampling fraction, 1:50) to capture stacks of confocal images at high magnification $(1000 \times)$. For analysis of axons with GAP-43, we examined another anterior cingulate area (A24), in addition to the neighboring A32, to increase the power of the analysis for the control cases in the anterior cingulate ( 3 control cases for A32 and 4 control cases for A24).

To reduce the fluorescent glare, we applied three-dimensional deconvolution algorithms to images before analysis with the aid of Autodeblur (Media Cybernetics). We used these image stacks to create threedimensional projections in ImageJ, which we viewed and resliced in the $x-, y$-, or $z$-axis, to decipher axon branches from crosses. Profile counts of axons with branches, or axons that expressed GAP-43, were obtained using Image and normalized by dividing with the total number of sampled axons in each case. We also assessed GAP-43 expression using an independent method by estimating the ratio of the surface area of GAP43-/NFP-200-positive axons to the total surface area of all axons labeled with NFP-200. Both high-resolution quantitative methods allowed accurate quantification of axons expressing GAP-43, while excluding unrelated signal in glia or non-neural tissue that might have concealed potential differences.

We estimated the thickness of the cortical gray matter of ACC (A32) in a series of $20-\mu \mathrm{m}$-thick coronal sections per case, including its divisions at the bottom of a sulcus, where the cortex is compressed, at the top of a gyrus, where the cortex is thick, and at relatively straight parts of the cortex, using ImageJ as previously described (Hilgetag and Barbas, 2006).

Stereological analysis at the EM. To determine the density of axons and the thickness of axons and myelin in the white matter, we sampled a volume of $\sim 1 \mathrm{~cm}^{3}$ below each prefrontal cortical area, with a systematic random sampling fraction of 1:1000 that yielded $>2000$ axons, per case, per area. We divided the white matter (as described in White matter segmentation, above) into a superficial part (closer to the gray matter) and a deep part.

High-resolution images of areas of interest were captured with a digital camera attached to the electron microscope (ES1000W, Gatan), imported in ImageJ, and calibrated. We estimated the overall density of axons at low magnification $(3300 \times)$ by dividing the number or the surface area of the axon profiles by the total surface area of the sampled region. We estimated the maximum inner and outer diameter as well as the thickness of the surrounding myelin sheath at high magnification $(10,000 \times)$. To minimize variability and test for errors due to sectioning, we measured axons that were perpendicular to the cutting plane and appeared cylindrical. We then repeated the analysis to include all axons by measuring the diameter perpendicular to the center of the maximum diameter of the axon profile. The two analyses yielded similar results and were combined.

Three-dimensional reconstruction and branching analysis. We followed axons in the superficial part of the white matter of ACC in three autistic and three control cases in long, uninterrupted series of $\sim 400$ ultrathin sections (thickness, $150 \mathrm{~nm}$ each). The volume examined was $\sim 600,000$ $\mu \mathrm{m}^{3}$. We viewed at least 200 axons per case at high magnification $(10,000 \times)$ and photographed them throughout their extent in the series using exhaustive sampling. High-resolution digital images were imported as a series in Reconstruct [http://www.bu.edu/neural (Fiala, 2005)] and aligned, as previously described (Zikopoulos and Barbas, 2006). Axons were traced, reconstructed in three dimensions, and their average diameters calculated. Traces of small, medium, large, and extralarge axons were color coded for easy visualization. We estimated the number of all branching points and the number of axons (by size) with branches in each series. Branches were reliably identified and associated with parent axons based on the continuity of the axoplasm, and the thinning or disappearance of myelin at the branching points.

Statistical analysis. Data were gathered blind to condition and cortical region. Random codes for cases and images were broken after completion of each part of the study. In all cases, data collection was performed by at least two investigators. Values obtained from the two independent measures were highly correlated (Pearson $r=0.97, p=0.001$ ). The samples were obtained from widely spaced sections (1 every 10) and fields of view through systematic random sampling to minimize the likelihood of sampling axons from the same parent branch. This sampling 

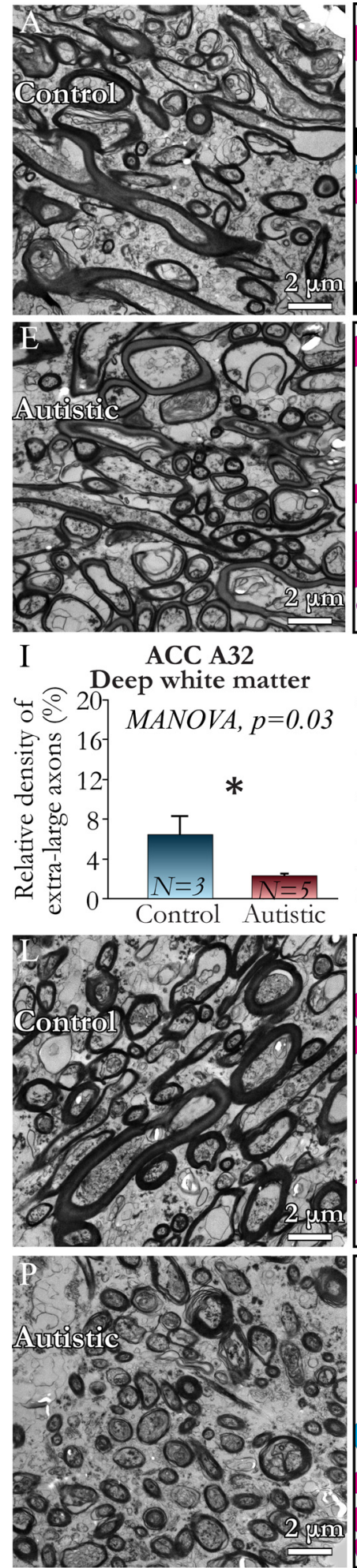
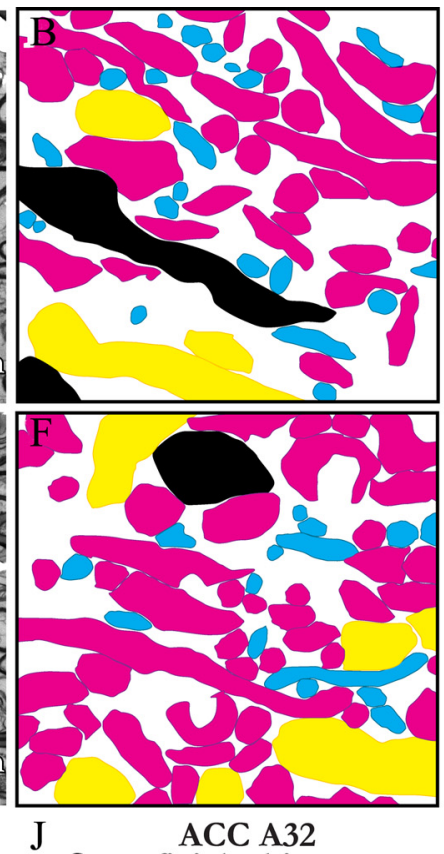

Superficial white matter

岸 1007 MANOVA, $p=0.01$
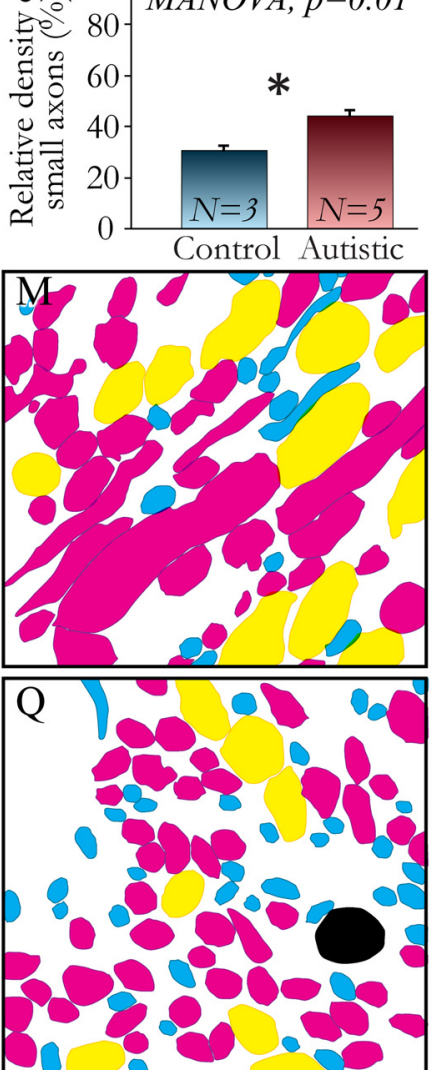
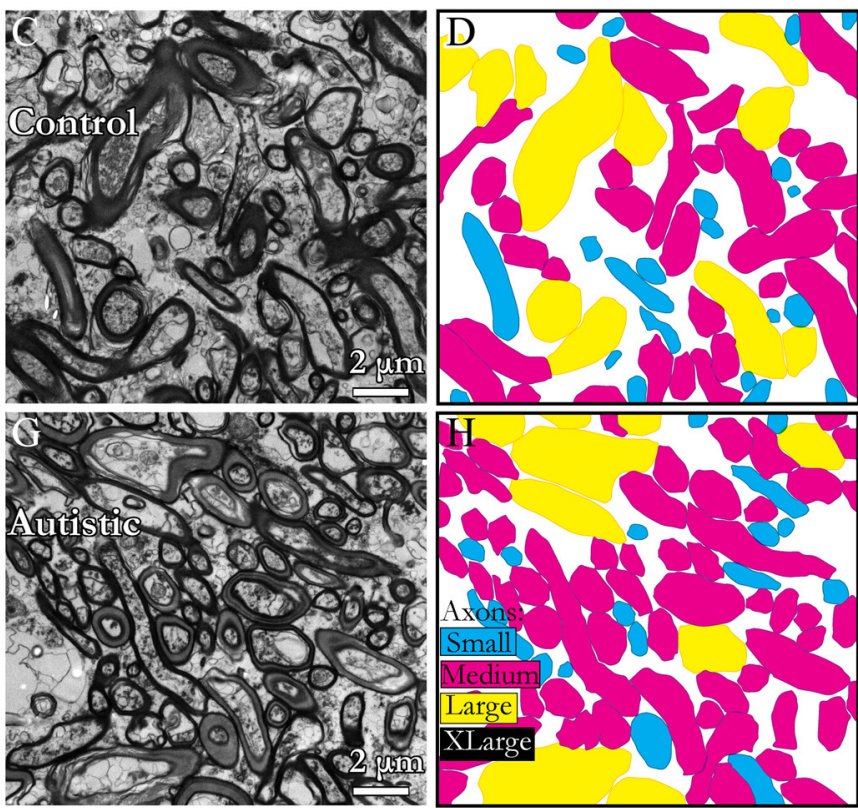

$\mathrm{K}$ Small axons $/ \mathrm{m}^{2}$

Medium axons $/ \mu \mathrm{m}^{2}$

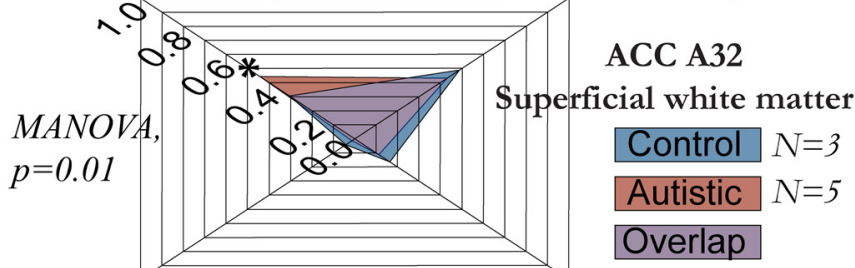

Extra-large axons $/ \mu \mathrm{m}^{2} \quad$ Large axons $/ \mu \mathrm{m}^{2}$
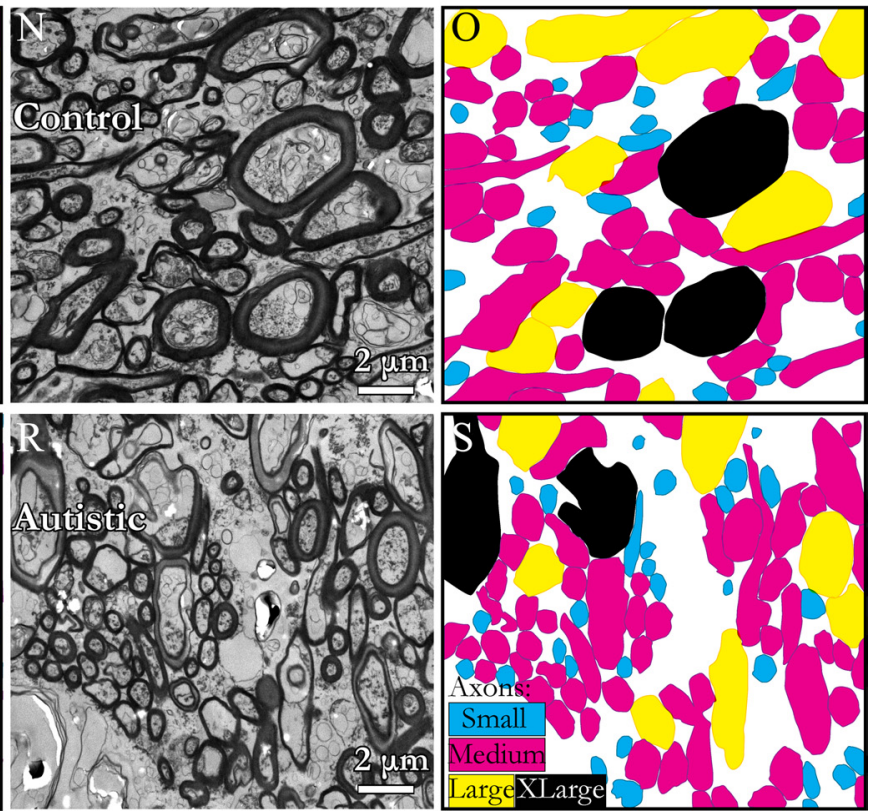

Figure 2. Altered axons below ACC in autism. $\boldsymbol{A}-\boldsymbol{H}$, Deep white matter: EM photomicrographs and respective plots (color coded) deeply below ACC (A32) in control and autistic cases show the distribution of small, medium, large and extra-large axons. $I$, The relative density of extra-large axons ( \pm SEM) is significantly lower ( ${ }^{*} p=0.03$ ) in autistic individuals. $J$, Superficial white matter: the relative density of small (thin) axons ( \pm SEM) just below ACC is significantly higher $\left({ }^{*} p=0.01\right.$ ) in the autistic cases. $K$, Same information as in $/$ plotted as a fingerprint of axons. $L-S$, Superficial white matter: EM photomicrographs and respective plots (color coded) in control and autistic cases show distribution of the four size groups of axons.

scheme and the fact that most axons branch very close to or after they enter the gray matter minimized the likelihood of counting segments of the same axon more than once.

Data distributions for continuous variables were not significantly different from normal, as determined by the Kolmogorov-Smirnov test, and thus allowed the use of parametric statistics. We initially used $\chi^{2}$ and Kolmogorov-Smirnov tests to examine axon size distributions and multiple linear regression analysis to examine correlations. Data were evaluated with Statistica (StatSoft), through scatter and frequency distribution plots and $\mathrm{K}$-means cluster analysis with parameters set to maximize 
initial between-cluster distances. We used MANOVA to test for differences among axon and neuron populations and densities. We then used post hoc analyses using Bonferroni/ Dunn (all means) corrections to identify possible differences between groups. For the axonbranching analysis, we used a two-tailed $t$ test. For all analyses, $p$ values $<0.05$ were taken as statistically significant.

We also used three different multivariate analyses to assess global similarities and dissimilarities of the white matter below prefrontal cortices based on all the ultrastructural features examined. We performed a discriminant analysis to identify experimental measures that minimize the overlap and clearly separate the distributions of individual data points belonging to different cortical areas for each case. We performed hierarchical cluster analysis (HCA) to group areas based on (dis)similarities in their parameter profiles. In this test, the relative similarity of areas is expressed as the distance between two branching points in a cluster tree diagram. Finally, we used nonmetric multidimensional scaling (NMDS) to arrange prefrontal areas in control and autistic cases in a low-dimensional (twodimensional) space based on the pairwise correlation (dis)similarities between areas. The relative proximity among items in an NMDS diagram represents their relative similarity. We performed NMDS using both the mean values for each area for the autistic and control cases to maximize their separation, as well as using the entire range of values for each case to take into account sample variability. HCA and NMDS analyses employed squared area (dis)similarity matrices derived from the normalized areal profiles by Pearson's correlation.

We also examined potential effects of sex, PMI, age at death, and other diagnoses (i.e., seizures) on all estimates for axon density, size, branching, expression of GAP-43, as well as neuronal and glial cell densities, using correlation analysis (supplemental Fig. $1 A-F$, available at www.jneurosci.org as supplemental material). In addition, we compared all estimated variables between and within control and autistic cases using MANCOVA with sex, PMI, age at death, and other diagnoses as the covariate and compared the results from this analysis with the MANOVA outcome.

The PMI for the control cases averaged ( \pm SEM) $17.5 \pm 1.5 \mathrm{~h}$, and for the autistic cases $39.8 \pm 16.9 \mathrm{~h}$ (Table 1 ). This number was significantly higher for the autistic cases because of case 4871 , which had a PMI of $99 \mathrm{~h}$. Without this case, the PMI for the autistic cases was comparable to the control cases $(25 \pm 4.8 \mathrm{~h})$. Examination of the structural integrity of the tissue and the quality of labeling revealed no differences between case 4871 and the other autistic cases at the light or confocal microscope. At the EM, the density of axons in case 4871 was not affected, but the membranes of some glial cells and small parts of the myelin surrounding some axons were compromised. As a result, we did not use this case for glial cell density estimates at the EM, and we sampled a much larger area to estimate axon and myelin diameter. The results obtained from case 4871 correlated well with measurements from the other autistic cases and were thus included in the analyses.

We performed additional statistical analyses to assess the generalizability of the results and to estimate whether the data could be used to accurately predict relationships between the estimated variables in
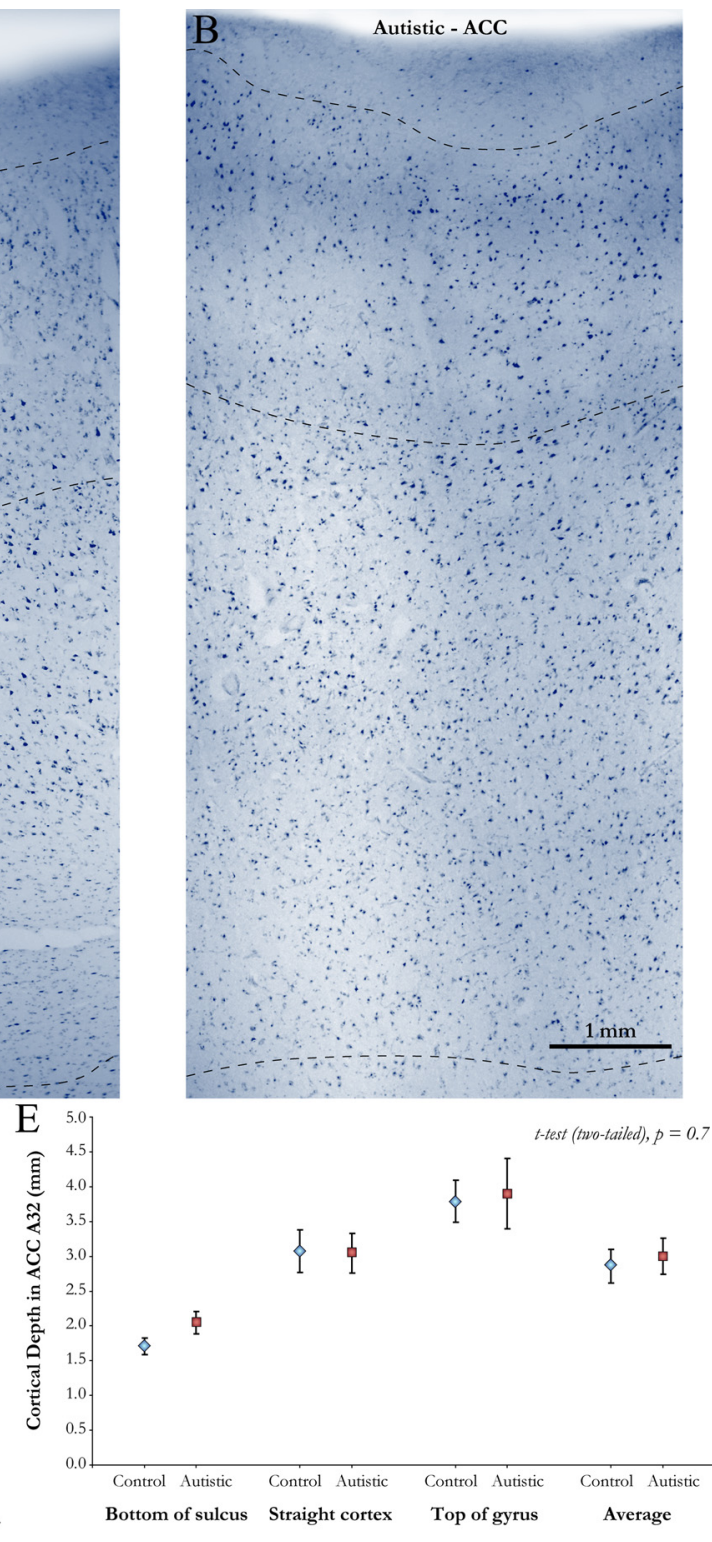

Bottom of sulcus Straight cortex Top of gyrus Average

Figure 3. Neuronal density and cortical thickness in ACC (A32) were not affected in the autistic cases. $A, B$, Photomontages of adjoining high-magnification images of Niss--stained coronal sections, from the pial surface to the white matter of ACC, in a contro C, Estimated overall neuronal density \pm SEM in ACC (A32) based on stereologic analysis. D, Plot of the laminar neuronal density \pm SEM in A32. $E$, Mean cortical gray matter thickness \pm SEM in sulcal, straight, and gyral parts of A32 and overall average thickness.

independent samples. To this effect, we used cross-validation techniques specifically designed to test the validity of the results and groupings derived from ANOVA, cluster and discriminant analyses, and NMDS. This method involved partitioning the sample of cases into $N=30$ complementary subsets, performing the analyses on $N-$ $1=29$ subsets, validating it on the other subset, and then repeating the process $N$ times. The $N=30$ sample subsets were determined so as to include all possible combinations of control and autistic cases in groups of three, which was a minimum requirement to perform all analyses (e.g., autistic group 1: cases 4541, 4871, and 5173; control group 1: cases 4786, 4981, and 5353; autistic group 2: cases 6232, 6677, and 5173; control group 2: cases 6004, 5353, and 4981). This method yields fits of predicted and actual data, which are acceptable if the root mean squared errors remain low. Finally, we used two complete datasets collected independently by two investigators and repeated all analyses using a repeated-measures design. 

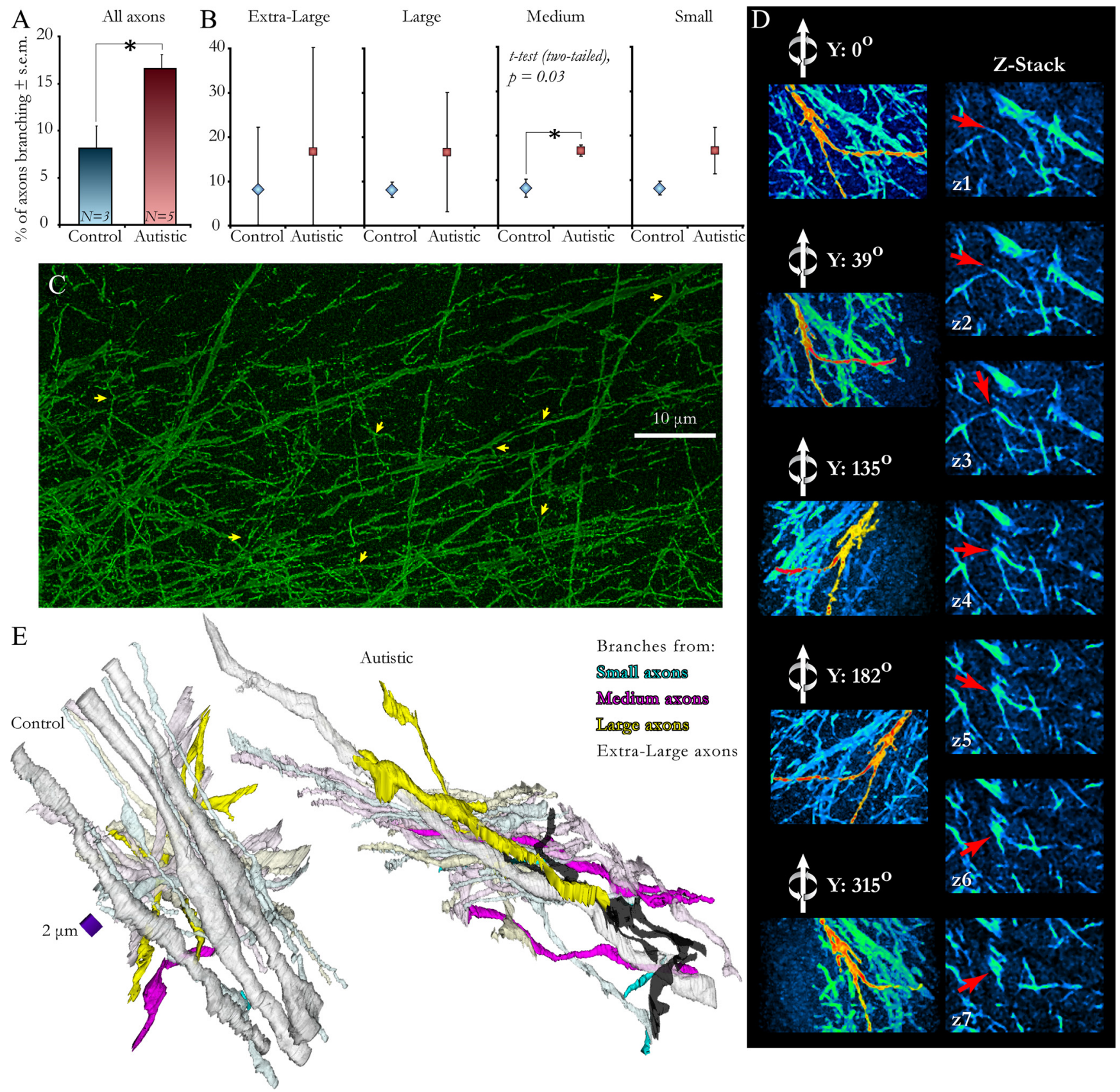

Figure 4. Increased branching of axons in the superficial white matter below ACC in autism. $\boldsymbol{A}$, Average $( \pm \mathrm{SEM})$ of all axons with branches is significantly higher $\left({ }^{*} p=0.03\right)$ in the autistic cases. $\boldsymbol{B}$, Average relative number of axons with branches ( \pm SEM) grouped by size. Medium-sized axons have significantly more branches $\left({ }^{*} p=0.03\right.$ ) in the autistic group. $\boldsymbol{C}$, Collapsed confocal image of myelinated axons (green) in an autistic case labeled with NFP-200. Yellow arrows show some branching points. D, Images from a three-dimensional confocal stack that was used for the branching analysis. The left column shows a $3 D$ projection of the confocal stack and rotation in the $y$-axis. A branching axon is pseudo-colored with orange/yellow hue for visualization. The right column (images z1-z7) shows the same axon (red arrow) in serial images $(0.4 \mu \mathrm{m}$ apart) from the $z$-axis confocal stack that was used to create the three-dimensional projections. E, Three-dimensional reconstruction of axons (gray) and their branches (color coded by size of the parent axon) from uninterrupted series of ultrathin sections ( $150 \mathrm{~nm}$ ) at the EM in a control (left) and an autistic case (right), which has more axons with branches.

\section{Results}

Myelinated axons made up $\sim 40 \%$ of the white matter in brain tissue from both autistic and control cases, resulting in an average density of 0.36 axons $/ \mu \mathrm{m}^{2}$, in agreement with previous studies in nonhuman primates (LaMantia and Rakic, 1990a,b, 1994). The rest of the white matter was occupied by glia, mainly oligodendrocytes.

\section{Axons below prefrontal areas are organized into four groups} by thickness

Overall axonal density between normal and autistic groups was similar below all prefrontal areas and parts of the white matter examined (supplemental Table 2, available at www.jneurosci.org as supplemental material). This finding is in agreement with previous reports indicating that the enlargement of the frontal white matter observed in children with autism is transient (Herbert et al., 2004).

However, axons vary in thickness, which affects their physiologic properties (Rushton, 1951; Wang et al., 2008), but their key features in prefrontal white matter and potential disruption in autism are unknown. We addressed this issue in brain tissue from both autistic ( $n=5,1$ female; 2000 axons/case) and control $(n=$ 4, 2 female; 2000 axons/case) individuals using EM. Myelinated 
axons varied in diameter, ranging from 0.1 to $7 \mu \mathrm{m}$ (axon thickness, inner diameter). The average diameter of axons in the superficial white matter was $0.8 \pm 0.02 \mu \mathrm{m}$, and in the deep white matter axons were slightly thicker $(0.9 \pm 0.02 \mu \mathrm{m})$. Similar axon sizes have been reported in other white matter regions of the primate brain, such as the corpus callosum, anterior commissure, and hippocampal commissure (LaMantia and Rakic, 1990a).

Cluster analysis of all axons segregated them into four groups based on inner diameter (without myelin), which were used for further comparisons (small, $<0.35 \mu \mathrm{m}$; medium, 0.35-0.69 $\mu \mathrm{m}$; large, $0.7-1.4 \mu \mathrm{m}$; and extra-large, $>1.4 \mu \mathrm{m} ; p=0.01)$. In all cases, most axons were small and medium in size (small, 36\%; medium, 46\%; large, 15\%; and extra-large, 3\%).

\section{Decreased long-range ACC axons in autism}

The relative position of axons within the white matter is an indicator of their termination in nearby or distant brain areas. The deep white matter includes mostly long-range pathways (Herbert et al., 2004; Hilgetag and Barbas, 2006; Petrides and Pandya, 2006, 2007; Schmahmann and Pandya, 2006; Sundaram et al., 2008). Previous studies have suggested that long-range corticocortical pathways that link frontal areas with other cortices are weak and disorganized in autism (Just et al., 2004, 2007; Courchesne and Pierce, 2005), but the cause is unknown. To address this issue, we measured the inner diameter of axons in the deep white matter. We found that the autistic group had significantly fewer extra-large axons only in area 32 of ACC (hereafter called ACC) compared with controls ( $p=0.03$ ) (Fig. $2 A-I$; supplemental Table 2, available at www.jneurosci.org as supplemental material). This group constituted a small proportion of all axons, but is within the range of densities of long-distance pathways, which are sparse in comparison with short-range pathways (Barbas, 1988). Nevertheless, long-distance pathways have considerable influence on the cortex. The prefrontal cortex, in particular, relies on sparse corticocortical pathways for all its sensory input.

To narrow down the list of possible long-range pathways affected in autism, we labeled axons with the calcium-binding proteins calbindin or parvalbumin, which are expressed by distinct classes of excitatory thalamic neurons that project to the cortex (Jones, 1998, 2007; Zikopoulos and Barbas, 2007). Quantitative analysis with EM showed no significant differences in the proportion of thalamo-cortical axons in autistic and control cases $(p=$ 0.23) (supplemental Fig. 2, available at www.jneurosci.org as supplemental material). This finding suggests that in autism there is reduction in other long-distance pathways. Among long-range pathways, the corticocortical connections are likely affected, in view of their functional disruption in autism (Just et al., 2004, 2007; Courchesne and Pierce, 2005). However, the involvement of other cortico-subcortical pathways, including cortical projections to the striatum or connections with the basal forebrain and brainstem cannot be excluded.

\section{Increased short- and medium-range ACC axons and branching in autism}

The superficial white matter contains mostly short- and medium-range axons that connect nearby areas, but also includes long-distance axons as they pass through to reach or exit the cortex. However, short- and medium-range pathways make up the bulk $(>80 \%)$ of all corticocortical connections (Barbas, 1988). We compared axons in the superficial white matter in autistic and control cases. Figure $2 J-S$ shows the results from this analysis and provides evidence that the density of small axons was significantly higher in the autistic group than in the control group, specifically in
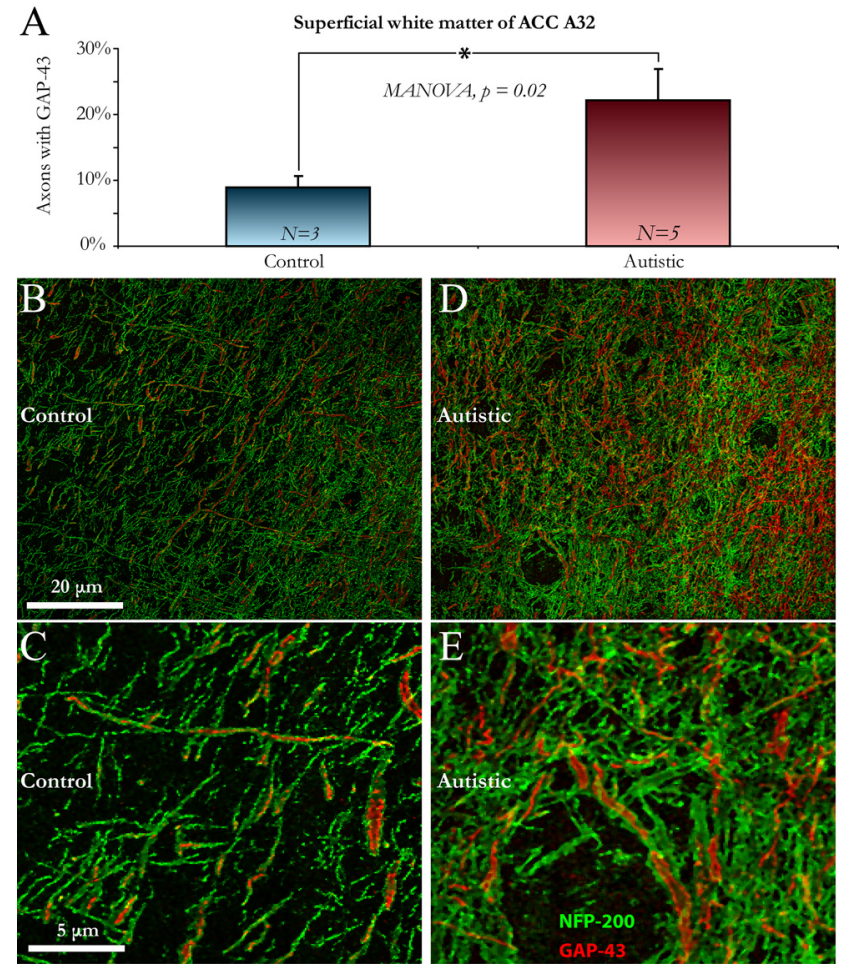

Figure 5. GAP-43 is elevated in the superficial white matter below ACC in autism. $\boldsymbol{A}$, Myelinated axons (percentage \pm SEM) labeled with the axon marker NFP-200 that also express GAP-43 is over twofold higher $\left({ }^{*} p=0.02\right)$ in autistic than in control cases. $\boldsymbol{B}, \boldsymbol{C}$, Control case: low $(\boldsymbol{B})$ and high ( $\boldsymbol{C}$ magnification of confocal images from double immunofluorescence show GAP-43 (red) in axons labeled with NFP-200 (green). Some myelinated axons contain GAP-43 in their axolemma, which is transported to axon terminals and branching points. $\boldsymbol{D}, \boldsymbol{E}$, Autistic case: low $(\boldsymbol{D})$ and high $(\boldsymbol{E})$ magnification of confocal images show increased number of axons with GAP-43.

the superficial white matter of ACC ( $p=0.01)$, but not below the other areas (supplemental Table 2, available at www.jneurosci.org as supplemental material).

We tested whether the higher density of small axons could be explained by differences in the density of the overlying neurons in ACC or the thickness of the cortex. There were no significant differences in the overall or laminar density of neurons in ACC in the brains of control (overall density, 32,536 $\pm 5,450 / \mathrm{mm}^{3}$ ) and autistic $\left(32,388 \pm 3,145 / \mathrm{mm}^{3}\right)$ cases (Fig. $\left.3 A-D\right)$, in overall cortical thickness (average thickness: control, $2.9 \pm 0.2 \mathrm{~mm}$; autistic, $3.0 \pm 0.3 ; p=0.7$ ), or in the segments of ACC separated into sulcal, straight cortex, or the top of the gyrus (Fig. $3 E$ ).

We reasoned that the higher density of small axons in the autistic cases must be due to increased branching of axons in the superficial white matter. To address this hypothesis, we followed and reconstructed axons $(>800)$ in three dimensions from large uninterrupted series of EM images. We found that the white matter below ACC had a significantly higher percentage of axons with branches in autistic compared with control cases only for medium axons (Fig. 4) ( $p=0.03)$, which give rise to small axons at bifurcations. The average number of branches per axon $( \pm$ SEM) in the superficial white matter below ACC was $2.6 \pm 1.4$ (control cases: $1 \pm 0.5$ for small axons; $1.8 \pm 0.1$ for medium axons; $4.2 \pm 3.1$ for large axons; $1 \pm 0.7$ for extra-large axons; autistic cases: $2.2 \pm 0.9$ for small axons; $3.2 \pm 1.8$ for medium axons; $3.2 \pm 1.6$ for large axons; $0.5 \pm 0.2$ for extra-large axons). Most points of bifurcation were unmyelinated or arose after thinning of the myelin [typically seen near the nodes of Ranvier 


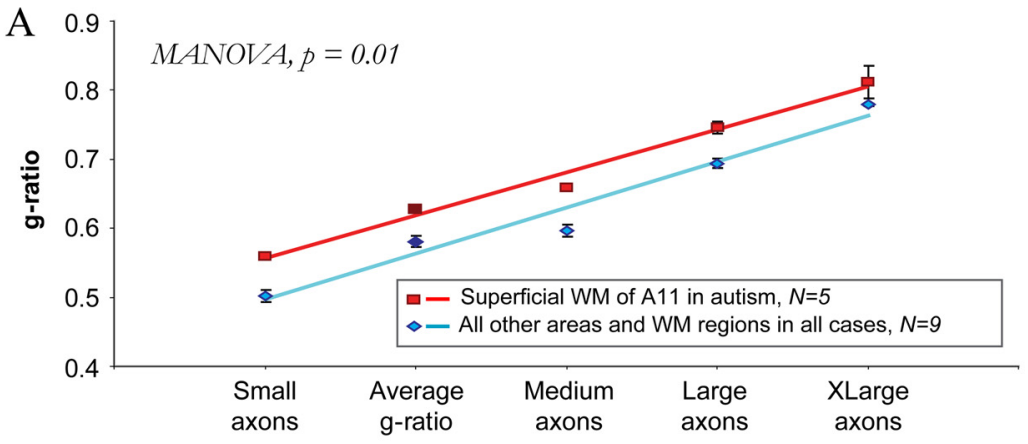

B
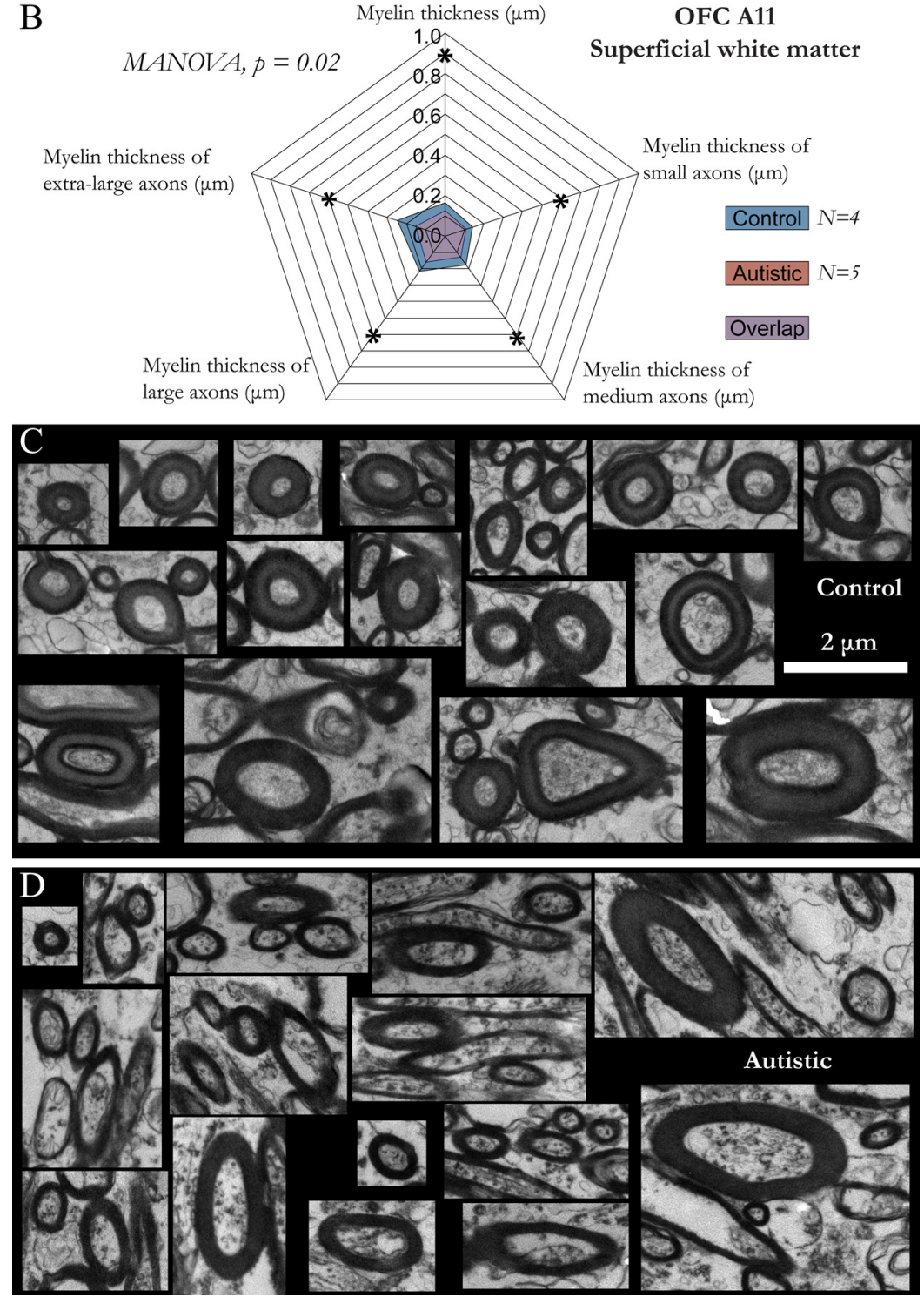

Figure 6. Decreased thickness of myelin in the superficial white matter below OFC (A11) in autism. A, G-ratio plot (inner/outer axon diameter \pm SEM) in all areas and cases examined. The decreased myelin thickness, found only in the superficial white matter below OFC, increased the g-ratio (red squares and line) above normal levels (blue diamonds and line). $\boldsymbol{B}$, Fingerprint plot shows that in $0 \mathrm{FC}$ there was significant $\left({ }^{*} p=0.01\right)$ overall decrease in the thickness of myelin in axons of all sizes. $\boldsymbol{C}, \boldsymbol{D}$, EM photomicrographs show differences in myelin thickness between control $(\boldsymbol{C})$ and autistic $(\boldsymbol{D})$ cases, apparent in all axon size groups.

(Peters et al., 1991)]. Axon branches were in most cases thinner than their parent axons, in agreement with previous reports (Ramon y Cajal, 1911; Peters et al., 1991; Schmitt et al., 2004).

Using an alternative method, we labeled myelinated axons with an antibody against a neurofilament protein (NFP-200) and deter- mined the proportion of axons that branched, using image stacks at the confocal microscope, which confirmed the EM results (Fig. 4C,D). Together, these data show an excess of axons that most likely course over short or medium distances. This finding is consistent with the hypothesis that prefrontal areas are overconnected in autism (Casanova, 2004; Courchesne and Pierce, 2005).

Increased GAP-43 below ACC in autism Supernumerary branching may be associated with increased axon production or decreased axon pruning occurring in the postnatal period (LaMantia and Rakic, 1990b). GAP-43 is expressed at high levels during rapid axon growth and is subsequently markedly reduced (Benowitz and Routtenberg, 1997). In the adult brain, GAP-43 is found in significant amounts only in association cortices, including the ACC, and at focal sites after brain injury (Benowitz and Routtenberg, 1997). Based on this evidence, we hypothesized that the high proportion of axons with branches immediately below ACC (A32) may reflect a high level of GAP-43 in autism. Immunohistochemical analysis of the superficial white matter showed a more than twofold increase in the proportion of axons that express GAP-43 only below ACC (A32) in autistic (22 $\pm 5 \%$ ) compared with control $(9 \pm 2 \%)$ cases $(p=0.02$ ) (Fig. 5), but not below the other areas (supplemental Fig. 3, available at www.jneurosci.org as supplemental material)

We then investigated whether other factors might have affected GAP-43 levels, such as medication, or comorbidity with schizophrenia, epilepsy, or depression, reported for three of the autistic cases. A correlation analysis did not reveal significant associations at the $95 \%$ confidence level; however, more autistic cases will need to be examined in future studies to fully address this issue. Moreover, analysis of a nearby cingulate area (A24) showed no difference in GAP-43 between autistic and control cases $(n=4$ control and 5 autistic cases) (supplemental Fig. 3, available at www.jneurosci.org as supplemental material). This evidence suggests that the increased proportion of axons that express GAP-43 in ACC A32 may be specific, though more areas must be studied to address this issue. It is not clear why neighboring cingulate areas 32 and 24 differ in the expression of GAP-43 in autism. The difference may be related to their developmental patterns, and in particular myelination, which is completed much earlier in A24 than in A32 (Flechsig, 1901). 

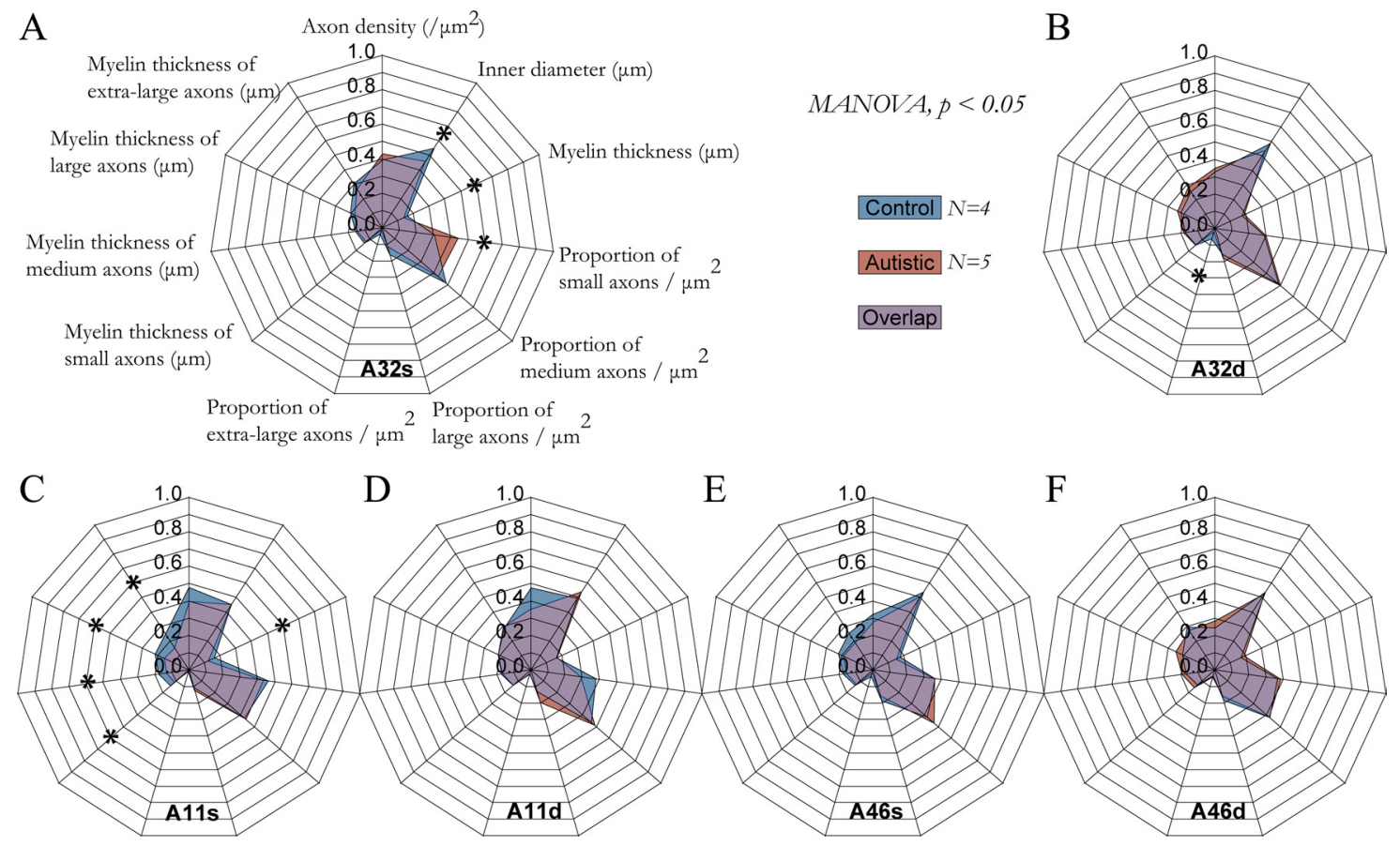

Figure 7. The structural features of axons and their density identify distinct prefrontal areas in control and autistic cases. $\boldsymbol{A}-\boldsymbol{F}$, Fingerprint plots of the superficial ( $s$ ) and deep (d) white matter below areas 32,11 , and $46 .{ }^{*}$ Significant differences between control and autistic cases $(p<0.05)$.

\section{Decreased thickness of myelin below OFC in autism}

We next investigated the thickness of myelin, which insulates axons and affects conduction velocity. We found a positive linear correlation between the thickness of axons and the thickness of their myelin sheath (Pearson $r=0.7, p<0.05$ ) (supplemental Fig. 4, available at www.jneurosci.org as supplemental material). This finding is consistent with the classic relationship of the inner to the outer diameter of axons, known as the g-ratio (Rushton, 1951). In all cases and areas, the g-ratio increased significantly with axon size (Fig. 6A), in agreement with recent studies (Paus and Toro, 2009).

Below ACC, overall myelin thickness was lower in autistic than in control cases, but the difference could be explained by the higher prevalence of thin axons, as predicted by the g-ratio (supplemental Table 2, available at www.jneurosci.org as supplemental material). In contrast, in the superficial white matter of OFC area 11 (hereafter called OFC) the myelin was significantly thinner in autistic than in control cases regardless of axon diameter (Fig. 6B-D). Moreover, below OFC there were no differences in the relative proportions of small, medium, large, and extra-large axons between autistic and control cases that could account for the overall thinner myelin. This evidence suggests that myelination per se is affected only below OFC among the areas studied. Consequently, the overall g-ratio ( \pm SEM) in OFC increased to $0.63 \pm 0.01$ from $0.58 \pm 0.01$ in all other cases and areas (Fig. $6 A$ ).

To determine whether the thinner myelin could be due to reduction in the number of oligodendrocytes, which myelinate central axons, we estimated their density in the white matter below OFC. We conducted independent stereologic analyses at the light microscope after immunohistochemical labeling for MOSP, and at the EM, based on the distinctive morphology of oligodendrocytes (supplemental Fig. 5, available at www. jneurosci.org as supplemental material). The two independent methods yielded similar data, and showed no significant differences $(p=0.53)$. The respective densities $( \pm$ SEM) were as fol- lows: at EM (oligodendrocyte profile counts $/ \mathrm{mm}^{2}$ ): control cases, $703 \pm 19$; autistic cases, $784 \pm 135$; and at LM (stereology, oligodendrocytes $/ \mathrm{mm}^{3}$ ): control cases, 137,203 \pm 24,397; autistic cases, 141,220 $\pm 71,877 ; p=0.53$ (supplemental Fig. 5, available at www.jneurosci.org as supplemental material).

\section{Interareal differences among prefrontal areas}

We then performed a discriminant analysis to identify the experimental measures that were most informative in distinguishing the white matter below specific prefrontal areas in control and autistic cases, and constructed detailed fingerprint diagrams (Fig. 7). All parameters used were highly characteristic for identifying individual areas in all cases, with the exception of overall axon density. Hierarchical cluster analysis showed a clear separation of ACC, OFC, and LPFC areas in control cases, which was less apparent in the autistic group. Independent nonmetric, multidimensional scaling analysis using both the mean values for each area for the autistic and control cases to maximize their separation, and using the entire range of values for each condition to take into account sample variability (Fig. 8; supplemental Fig. $1 G-K$, available at www.jneurosci.org as supplemental material), corroborated these results and showed that the axons below lateral prefrontal A46 (LPFC) were not affected in autism. However, the white matter below ACC and OFC had altered characteristics in autism and were more similar to each other than to their respective controls.

The significant increase in the number of small axons below ACC in the autistic cases changed the relationship between the prefrontal areas examined and revealed additional differences (Fig. 9). In control cases, OFC had a higher proportion of small axons than ACC (Fig. 9A), and a lower proportion of large axons than LPFC (Fig. 9B). In turn, ACC had a higher proportion of medium axons than LPFC (Fig. 9C). These interareal differences were not apparent in the autistic group, which had more small axons in the deep white matter of LPFC compared with OFC. This evidence indicates that the relationships among these pre- 


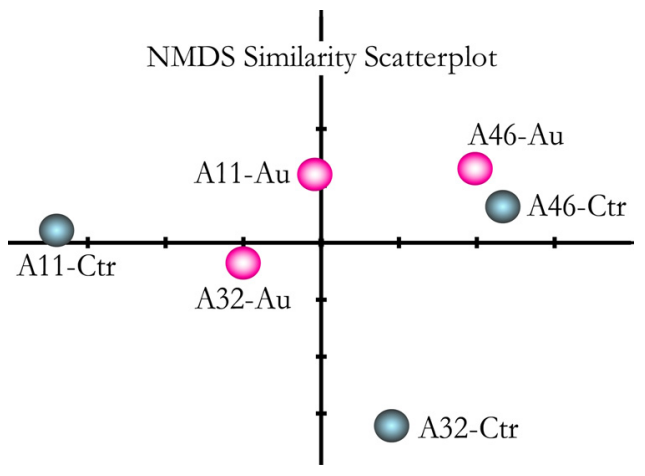

Figure 8. Profile of prefrontal areas based on their axon features. NMDS based on all measures of axon features shows a clear separation of three prefrontal areas in controls (blue), and an altered relationship in the autistic cases (red). Alienation coefficient $=0.039$.

frontal areas are altered in autism, suggesting widespread repercussions on neural communication (Bauman and Kemper, 2005; Loveland et al., 2008).

\section{Cross-validation analyses of the results}

We performed a series of additional statistical analyses to assess the robustness and generalizability of the findings. Correlation analysis showed no potential effects of sex, PMI, age at death, and other diagnoses (i.e., seizures) on all estimates for axon density, size, branching, expression of GAP-43, as well as neuronal and glial cell densities at the 95\% confidence level (supplemental Fig. $1 A-F$, available at www.jneurosci.org as supplemental material). The only significant correlation was between sex and the density of thalamocortical axons, where the three female cases (one autistic and two controls) had higher values than the male cases. In addition, we compared all estimated variables between and within control and autistic cases using MANCOVA with sex, $\mathrm{PMI}$, age at death, and other diagnoses as the covariate, and detected the same changes as with MANOVA with no significant correlations with the covariant variables.

In view of evidence for significant changes in the structure, neurochemistry, and function of the cortex with age, both in autistic and in typically developing individuals, we selected postmortem tissue from cases that were closely matched regarding age at death, and ranged from 30 to 44 years. In contrast to the major neural changes that take place during childhood, teenage years, and late adulthood, there is no evidence for significant changes in the density of neurons, axons, and synapses as well as in the myelination of axons within this age range in autistic or typically developing individuals (for review, see Yakovlev and Lecours, 1967; Bauman and Kemper, 2005; Redcay and Courchesne, 2005; Amaral et al., 2008). In agreement with these studies, we found no significant correlation of age at death with any of the estimated parameters (supplemental Fig. $1 A-F$, available at www.jneurosci.org as supplemental material).

The cross-validation tests conducted to estimate whether the data could be used to accurately predict relationships between the estimated variables in independent samples were specifically designed to test the validity of results and groupings derived from ANOVA, cluster and discriminant analyses, and NMDS. These tests, which were performed on all possible combinations of control and autistic cases in groups of three, yielded highly accurate fits of predicted and actual data with low root mean squared errors $(\leq 0.01)$, and concurred with the previous analyses. Finally, we used two complete datasets collected independently by

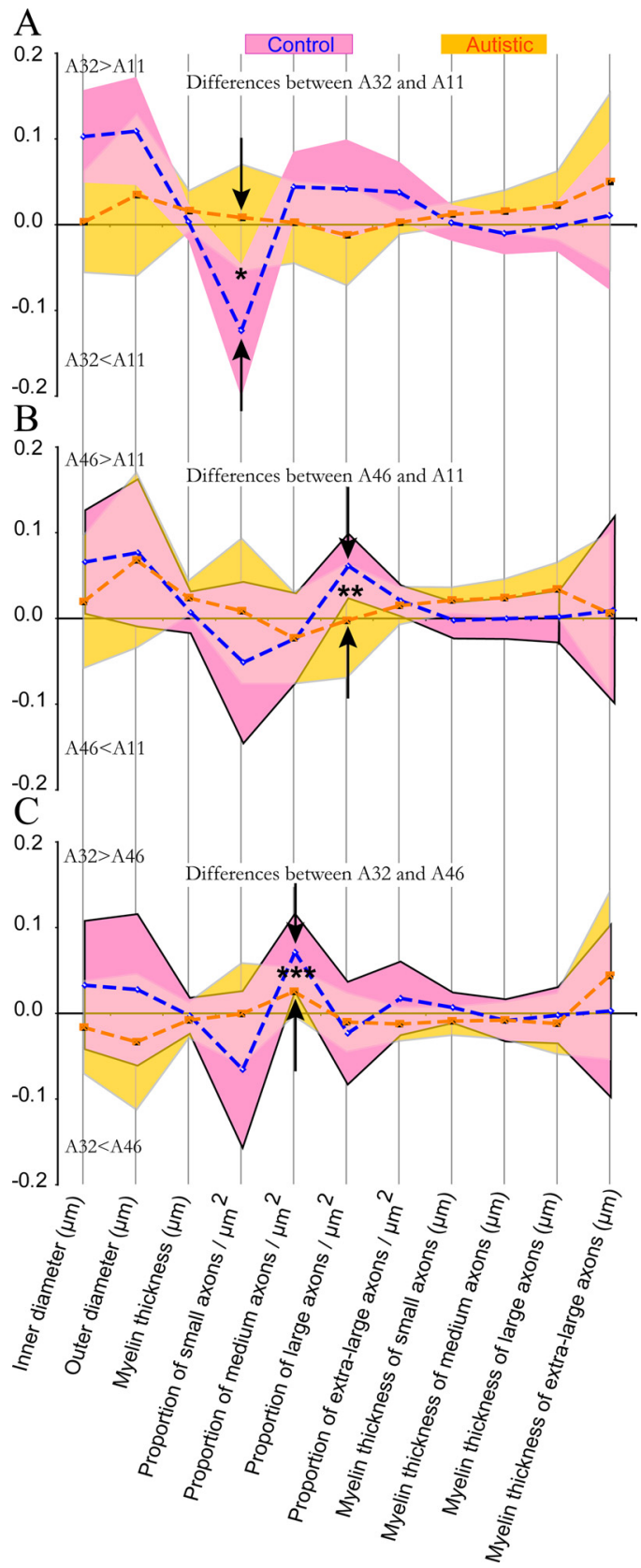

Figure 9. Changes in structural features of axons and density alter the relationship of ACC, $\mathrm{OFC}$, and LPFC in autism. Interareal differences were assessed by subtraction of corresponding normalized values for each pair of areas. $A$, Differences between A32 (ACC) and A11 (OFC); positive numbers indicate higher values for $A 32$, and negative numbers indicate higher values for A11. B, Differences between A46 (LPFC) and A11; positive numbers indicate higher values for A46, and negative numbers indicate higher values for A11. C, Differences between A32 and A46; positive numbers indicate higher values for $A 32$, and negative numbers indicate higher values for A46. Blue dotted line and magenta-shaded area indicate mean and range for the control cases. Orange dotted line and yellow-shaded area show mean and range for autistic cases. Asterisks indicate significant differences between control and autistic cases $(p<0.05)$.

two investigators and performed all analyses in a repeatedmeasures design, which also yielded similar results.

The robustness of the results was strengthened by the fact that variability in all estimated parameters was low and the observed differences were found in all cases with no exception (supplemental Figs. 6, 7, available at www.jneurosci.org as supplemental material). 


\section{Discussion}

\section{Disruption of prefrontal networks in autism}

Previous studies have suggested that the fundamental defect in autism is at the synapse (Südhof, 2008; Bourgeron, 2009). Our findings show physical changes in single axons below prefrontal areas in autism that likely affect synaptic function. The changes in axons were found in all autistic cases regardless of the presence or absence of epilepsy or mental retardation (Table 1; supplemental Figs. 6, 7, available at www.jneurosci.org as supplemental material). These findings suggest a fundamental autism phenotype in axons that make up the brain's communication system.

The high density of thin axons below ACC is consistent with studies suggesting excessive short-range connectivity in autism (Courchesne and Pierce, 2005). The presence of supernumerary axons likely accounts for the increased cortical folding in the frontal lobe in autism, consistent with the hypothesis that tension exerted by corticocortical connections is a significant factor in shaping the gyrencephalic cerebral cortex (Van Essen, 1997; Hilgetag and Barbas, 2006). Diseases of developmental origin lead to atypical folds (Levitt et al., 2003; Nordahl et al., 2007), suggesting abnormal connectivity.

The areas studied are robustly interconnected, and have a key role in emotions, attentional mechanisms, and executive control (Barbas, 2000), in processes that are severely affected in autism. In particular, the OFC has an overview of the sensory environment and, through robust connections with the amygdala, participates in the process of assessing the emotional significance of events (Barbas and Zikopoulos, 2006). The change in the optimal relationship between axon diameter and myelin in OFC, and the reduction of neurons in the amygdala in autism (Schumann and Amaral, 2006), provide the anatomic basis for disrupted transmission of signals for emotions (Bachevalier and Loveland, 2006; Loveland et al., 2008). Specifically, the increase in the g-ratio of axons above the optimal average value $(\sim 0.6)$ suggests suboptimal conduction velocity (Rushton, 1951; Paus and Toro, 2009), and these factors have been linked to cytoskeletal defects that affect cell metabolism and neurotransmission (Paus and Toro, 2009).

On the other hand, the ACC has a role in attentional processes and has the most widespread connections within the prefrontal cortex (Barbas et al., 1999). Through these robust connections, the ACC may affect function in LPFC, which has a key role in cognition. For example, when excitatory axons from ACC form synapses with inhibitory neurons in LPFC they target preferentially calbindin-inhibitory neurons (Medalla and Barbas, 2009), which have modulatory effects on pyramidal neurons, increasing the signal-to-noise ratio (Wang et al., 2004). These synaptic specializations suggest that ACC can reduce noise in LPFC, and facilitate holding attention on a task. The exuberance of thin axons in ACC in autism suggests a potential exaggeration of this mechanism, consistent with atypical LPFC activation in autism reported in functional imaging studies (Luna et al., 2002).
The ACC develops early in ontogeny in nonhuman primates (Rakic, 2002), suggesting early engagement of synaptic sites by its axons. Exuberance of thin axons that course over short or medium distances in autism may lead to occupation of sites normally available to the considerably sparser long-distance pathways. The latter are at a competitive disadvantage, not only because they develop later, but also because they need additional time to extend long axons to form synapses in the prefrontal cortex. Reduction in the strength of long-distance pathways in autism may thus be secondary to the excessive short-range connections of ACC. This connectivity bias may help explain why individuals with autism do not adequately shift attention when necessary, and engage in repetitive and inflexible behavior (Luna et al., 2002; Thakkar et al., 2008; Minshew and Keller, 2010).

In contrast to ACC, in OFC there were no differences in the composition of the four size groups of axons, but the myelin was overall thinner. What underlies these seemingly disparate changes in axons in ACC and OFC? The varied genetic mutations that confer susceptibility to autism affect, in general, aspects of development (Walsh et al., 2008; Weiss et al., 2009). The interplay of developmental events may help explain all the observed changes in axons, as summarized in Figure 10. Normal development is initiated with neurogenesis and migration followed by axon elongation in an environment enriched with GAP-43. Myelination occurs after axons grow, and extends well beyond the second year of life (Yakovlev and Lecours, 1967) and into adulthood (Paus et al., 1999). Once myelination is initiated, signals from myelin proteins help stabilize axons by inhibiting GAP-43 synthesis and halting axon growth (Kapfhammer and Schwab, 1994). Importantly, the inhibitory effects are reciprocal, so that GAP-43 exerts inhibitory effects on myelin (Kapfhammer and Schwab, 1994).

In nonhuman primates, neuronal migration in ACC is completed first among the areas studied (Rakic, 2002), but myelination occurs considerably later in A32 (Flechsig, 1901; Von Bonin, 
1950), suggesting that the ACC has prolonged exposure to GAP-43 in an environment that is permissive for axon growth. Moreover, within the white matter, GAP-43 is highest in its outer border, which is closest to the cortex, consistent with its rapid transport to axon terminals (Benowitz and Routtenberg, 1997). The significant increase in GAP-43 in the superficial white matter of ACC in autism is consistent with the exuberance of short- and medium-range axons. The assumption in our model, that GAP-43 in ACC A32 is elevated during development, is based on the increased levels seen in adults with autism (this study) and findings that the frontal white matter is enlarged in children with autism (Herbert et al., 2004). Further, our finding that myelin thickness in ACC was unaffected in autism can be explained by the fact that ACC axons in A32 myelinate very late (Flechsig, 1901), when GAP-43 levels are lower. On the other hand, OFC (area 11) develops after ACC (Rakic, 2002), but its myelination begins earlier than in ACC (Flechsig, 1901). The level of GAP-43 in OFC at the time of myelination onset is unknown, since our analysis was of adult brains. However, because the interval from cell migration to myelination is shorter in OFC than in ACC, even a small elevation in GAP-43 expression may be sufficient to retard myelin growth without causing excessive branching of axons, as seen here. Finally, LPFC (area 46) develops after ACC and OFC (Rakic, 2002) and myelinates very late (Flechsig, 1901; Von Bonin, 1950), so neither axon growth nor myelination is affected in autism. This model suggests that the distinct abnormalities in ACC A32 and OFC A11 in the autistic cases are linked and traced to a common developmental disturbance that affects the onset and perhaps the duration of expression of GAP-43 and its interaction with myelin.

Neuronal migration appears to be intact in autism, since neither neuronal density nor cortical depth was affected, at least in the parts of the three areas studied (supplemental data, available at www.jneurosci.org as supplemental material, cytoarchitecture of ACC, OFC, and LPFC). Our data suggest that the insult occurs later, when axons connect with other areas in the presence of high GAP-43 expression and possibly other growth factors, which may remain elevated in adulthood in response to inflammation (Vargas et al., 2005). The associated signaling pathways need to be investigated for therapeutic interventions in autism.

GAP-43 is up-regulated by a variety of external factors as well, including estrogenic agents that disrupt endocrine function, such as bisphenol A, used for lining plastic food and drink containers, linoleic acids found in some oils, and by immunosuppressive and psychiatric drugs used for a variety of common disorders, including psoriasis, asthma, rheumatoid arthritis, depression, and anxiety (Wong et al., 1989; Jyonouchi et al., 2001; Granda et al., 2003; Croen et al., 2005; Østensen et al., 2006; Sairanen et al., 2007; Brown, 2009; Nguyen et al., 2009). Several of these substances came into heavy use in the early 1980s at a time when the prevalence of autism began to rise (Blaxill, 2004). Epidemiological studies are necessary to investigate whether these events are merely coincident or whether the cumulative effects of dietary factors and drugs change the uterine and postnatal environment, and perturb the expression of factors implicated in axon growth and guidance in autism.

\section{References}

Amaral DG, Schumann CM, Nordahl CW (2008) Neuroanatomy of autism. Trends Neurosci 31:137-145.

Bachevalier J, Loveland KA (2006) The orbitofrontal-amygdala circuit and self-regulation of social-emotional behavior in autism. Neurosci Biobehav Rev 30:97-117.

Barbas H (1988) Anatomic organization of basoventral and mediodorsal visual recipient prefrontal regions in the rhesus monkey. J Comp Neurol 276:313-342.

Barbas H (2000) Complementary role of prefrontal cortical regions in cognition, memory and emotion in primates. Adv Neurol 84:87-110.

Barbas H, Pandya DN (1989) Architecture and intrinsic connections of the prefrontal cortex in the rhesus monkey. J Comp Neurol 286:353-375.

Barbas H, Zikopoulos B (2006) Sequential and parallel circuits for emotional processing in primate orbitofrontal cortex. In: The orbitofrontal cortex (Zald D, Rauch S, eds), pp 57-91. New York: Oxford UP.

Barbas H, Ghashghaei H, Dombrowski SM, Rempel-Clower NL (1999) Medial prefrontal cortices are unified by common connections with superior temporal cortices and distinguished by input from memory-related areas in the rhesus monkey. J Comp Neurol 410:343-367.

Barnea-Goraly N, Kwon H, Menon V, Eliez S, Lotspeich L, Reiss AL (2004) White matter structure in autism: preliminary evidence from diffusion tensor imaging. Biol Psychiatry 55:323-326.

Bauman ML, Kemper TL (2005) Neuroanatomic observations of the brain in autism: a review and future directions. Int J Dev Neurosci 23:183-187.

Benowitz LI, Routtenberg A (1997) GAP-43: an intrinsic determinant of neuronal development and plasticity. Trends Neurosci 20:84-91.

Blaxill MF (2004) What's going on? The question of time trends in autism. Public Health Rep 119:536-551.

Bourgeron T (2009) A synaptic trek to autism. Curr Opin Neurobiol 19:231-234.

Brown JS Jr (2009) Effects of bisphenol-A and other endocrine disruptors compared with abnormalities of schizophrenia: an endocrine-disruption theory of schizophrenia. Schizophr Bull 35:256-278.

Buxhoeveden DP, Semendeferi K, Buckwalter J, Schenker N, Switzer R, Courchesne E (2006) Reduced minicolumns in the frontal cortex of patients with autism. Neuropathol Appl Neurobiol 32:483-491.

Casanova MF (2004) White matter volume increase and minicolumns in autism. Ann Neurol 56:453.

Courchesne E, Pierce K (2005) Why the frontal cortex in autism might be talking only to itself: local over-connectivity but long-distance disconnection. Curr Opin Neurobiol 15:225-230.

Croen LA, Grether JK, Yoshida CK, Odouli R, Van de Water J (2005) Maternal autoimmune diseases, asthma and allergies, and childhood autism spectrum disorders: a case-control study. Arch Pediatr Adolesc Med 159:151-157.

Dombrowski SM, Hilgetag CC, Barbas H (2001) Quantitative architecture distinguishes prefrontal cortical systems in the rhesus monkey. Cereb Cortex 11:975-988.

Fiala JC (2005) Reconstruct: a free editor for serial section microscopy. J Microsc 218:52-61.

Flechsig P (1901) Developmental (myelogenetic) localisation of the cerebral cortex in the human subject. Lancet 1027-1029.

Girgis RR, Minshew NJ, Melhem NM, Nutche JJ, Keshavan MS, Hardan AY (2007) Volumetric alterations of the orbitofrontal cortex in autism. Prog Neuropsychopharmacol Biol Psychiatry 31:41-45.

Glessner JT, Wang K, Cai G, Korvatska O, Kim CE, Wood S, Zhang H, Estes A, Brune CW, Bradfield JP, Imielinski M, Frackelton EC, Reichert J, Crawford EL, Munson J, Sleiman PM, Chiavacci R, Annaiah K, Thomas $\mathrm{K}$, Hou C, et al. (2009) Autism genome-wide copy number variation reveals ubiquitin and neuronal genes. Nature 459:569-573.

Granda B, Tabernero A, Tello V, Medina JM (2003) Oleic acid induces GAP-43 expression through a protein kinase $\mathrm{C}$-mediated mechanism that is independent of NGF but synergistic with NT-3 and NT-4/5. Brain Res 988:1-8.

Griebling J, Minshew NJ, Bodner K, Libove R, Bansal R, Konasale P, Keshavan MS, Hardan A (2010) Dorsolateral prefrontal cortex magnetic resonance imaging measurements and cognitive performance in autism. J Child Neurol 25:856-863.

Gundersen HJ (1986) Stereology of arbitrary particles. A review of unbiased number and size estimators and the presentation of some new ones, in memory of William R. Thompson. J Microsc 143:3-45.

Hardan AY, Girgis RR, Lacerda AL, Yorbik O, Kilpatrick M, Keshavan MS, Minshew NJ (2006) Magnetic resonance imaging study of the orbitofrontal cortex in autism. J Child Neurol 21:866-871.

Herbert MR, Ziegler DA, Makris N, Filipek PA, Kemper TL, Normandin JJ, Sanders HA, Kennedy DN, Caviness VS Jr (2004) Localization of white matter volume increase in autism and developmental language disorder. Ann Neurol 55:530-540. 
Hilgetag CC, Barbas H (2006) Role of mechanical factors in the morphology of the primate cerebral cortex. PLoS Comput Biol 2:e22.

Howard CV, Reed MG (1998) Unbiased stereology, three-dimensional measurement in microscopy. Oxford: BIOS Scientific Publishers.

Jones EG (1998) Viewpoint: the core and matrix of thalamic organization. Neuroscience 85:331-345.

Jones EG (2007) The thalamus. New York: Cambridge UP.

Just MA, Cherkassky VL, Keller TA, Minshew NJ (2004) Cortical activation and synchronization during sentence comprehension in high-functioning autism: evidence of underconnectivity. Brain 127:1811-1821.

Just MA, Cherkassky VL, Keller TA, Kana RK, Minshew NJ (2007) Functional and anatomical cortical underconnectivity in autism: evidence from an fMRI study of an executive function task and corpus callosum morphometry. Cereb Cortex 17:951-961.

Jyonouchi H, Sun S, Le H (2001) Proinflammatory and regulatory cytokine production associated with innate and adaptive immune responses in children with autism spectrum disorders and developmental regression. J Neuroimmunol 120:170-179.

Kana RK, Keller TA, Cherkassky VL, Minshew NJ, Just MA (2006) Sentence comprehension in autism: thinking in pictures with decreased functional connectivity. Brain 129:2484-2493.

Kanazir S, Ruzdijic S, Vukosavic S, Ivkovic S, Milosevic A, Zecevic N, Rakic L (1996) GAP-43 mRNA expression in early development of human nervous system. Brain Res Mol Brain Res 38:145-155.

Kapfhammer JP, Schwab ME (1994) Inverse patterns of myelination and GAP-43 expression in the adult CNS: neurite growth inhibitors as regulators of neuronal plasticity? J Comp Neurol 340:194-206.

Keller TA, Kana RK, Just MA (2007) A developmental study of the structural integrity of white matter in autism. Neuroreport 18:23-27.

Koshino H, Kana RK, Keller TA, Cherkassky VL, Minshew NJ, Just MA (2008) fMRI investigation of working memory for faces in autism: visual coding and underconnectivity with frontal areas. Cereb Cortex 18:289-300.

LaMantia AS, Rakic P (1990a) Cytological and quantitative characteristics of four cerebral commissures in the rhesus monkey. J Comp Neurol 291:520-537.

LaMantia AS, Rakic P (1990b) Axon overproduction and elimination in the corpus callosum of the developing rhesus monkey. J Neurosci 10:2156-2175.

LaMantia AS, Rakic P (1994) Axon overproduction and elimination in the anterior commissure of the developing rhesus monkey. J Comp Neurol 340:328-336.

Levitt JG, Blanton RE, Smalley S, Thompson PM, Guthrie D, McCracken JT, Sadoun T, Heinichen L, Toga AW (2003) Cortical sulcal maps in autism. Cereb Cortex 13:728-735.

Loveland KA, Bachevalier J, Pearson DA, Lane DM (2008) Fronto-limbic functioning in children and adolescents with and without autism. Neuropsychologia 46:49-62.

Luna B, Minshew NJ, Garver KE, Lazar NA, Thulborn KR, Eddy WF, Sweeney JA (2002) Neocortical system abnormalities in autism: an fMRI study of spatial working memory. Neurology 59:834-840.

Medalla M, Barbas H (2009) Synapses with inhibitory neurons differentiate anterior cingulate from dorsolateral prefrontal pathways associated with cognitive control. Neuron 61:609-620.

Meyer JW, Makris N, Bates JF, Caviness VS, Kennedy DN (1999) MRIbased topographic parcellation of human cerebral white matter. Neuroimage 9:1-17.

Miguel-Hidalgo JJ, Overholser JC, Meltzer HY, Stockmeier CA, Rajkowska G (2006) Reduced glial and neuronal packing density in the orbitofrontal cortex in alcohol dependence and its relationship with suicide and duration of alcohol dependence. Alcohol Clin Exp Res 30:1845-1855.

Milosevic A, Kanazir S, Zecevic N (1995) Immunocytochemical localization of growth-associated protein GAP-43 in early human development. Brain Res Dev Brain Res 84:282-286.

Minshew NJ, Keller TA (2010) The nature of brain dysfunction in autism: functional brain imaging studies. Curr Opin Neurol 23:124-130.

Minshew NJ, Williams DL (2007) The new neurobiology of autism: cortex, connectivity, and neuronal organization. Arch Neurol 64:945-950.

Morrow EM, Yoo SY, Flavell SW, Kim TK, Lin Y, Hill RS, Mukaddes NM, Balkhy S, Gascon G, Hashmi A, Al-Saad S, Ware J, Joseph RM, Greenblatt R, Gleason D, Ertelt JA, Apse KA, Bodell A, Partlow JN, Barry B, et al.
(2008) Identifying autism loci and genes by tracing recent shared ancestry. Science 321:218-223.

Nguyen T, Lindner R, Tedeschi A, Forsberg K, Green A, Wuttke A, Gaub P, Di Giovanni S (2009) NFAT-3 is a transcriptional repressor of the growthassociated protein 43 during neuronal maturation. J Biol Chem 284:18816-18823.

Nordahl CW, Dierker D, Mostafavi I, Schumann CM, Rivera SM, Amaral DG, Van Essen DC (2007) Cortical folding abnormalities in autism revealed by surface-based morphometry. J Neurosci 27:11725-11735.

Oishi T, Higo N, Umino Y, Matsuda K, Hayashi M (1998) Development of GAP-43 mRNA in the macaque cerebral cortex. Brain Res Dev Brain Res 109:87-97.

Østensen M, Khamashta M, Lockshin M, Parke A, Brucato A, Carp H, Doria A, Rai R, Meroni P, Cetin I, Derksen R, Branch W, Motta M, Gordon C, Ruiz-Irastorza G, Spinillo A, Friedman D, Cimaz R, Czeizel A, Piette JC, et al (2006) Anti-inflammatory and immunosuppressive drugs and reproduction. Arthritis Res Ther 8:209.

Paus T, Toro R (2009) Could sex differences in white matter be explained by g ratio? Front Neuroanat 3:14.

Paus T, Zijdenbos A, Worsley K, Collins DL, Blumenthal J, Giedd JN, Rapoport JL, Evans AC (1999) Structural maturation of neural pathways in children and adolescents: in vivo study. Science 283:1908-1911.

Peters A, Palay SL, Webster HD (1991) The fine structure of the nervous system. Neurons and their supporting cells. New York: Oxford UP.

Petrides M, Pandya DN (2006) Efferent association pathways originating in the caudal prefrontal cortex in the macaque monkey. J Comp Neurol 498:227-251.

Petrides M, Pandya DN (2007) Efferent association pathways from the rostral prefrontal cortex in the macaque monkey. J Neurosci 27:11573-11586.

Rakic P (2002) Neurogenesis in adult primate neocortex: an evaluation of the evidence. Nat Rev Neurosci 3:65-71.

Ramon y Cajal S (1911) Histologie du systeme nerveux de l' homme et des vertebres. Paris: Maloine.

Redcay E, Courchesne E (2005) When is the brain enlarged in autism? A meta-analysis of all brain size reports. Biol Psychiatry 58:1-9.

Rubenstein JL, Merzenich MM (2003) Model of autism: increased ratio of excitation/inhibition in key neural systems. Genes Brain Behav 2:255-267.

Rushton WAH (1951) A theory of the effects of fibre size in medullated nerve. J Physiol 101-122.

Sairanen M, O’Leary OF, Knuuttila JE, Castrén E (2007) Chronic antidepressant treatment selectively increases expression of plasticity-related proteins in the hippocampus and medial prefrontal cortex of the rat. Neuroscience 144:368-374.

Schmahmann JD, Pandya DN (2006) Fiber pathways of the brain. New York: Oxford UP.

Schmitt O, Pakura M, Aach T, Hömke L, Böhme M, Bock S, Preusse S (2004) Analysis of nerve fibers and their distribution in histologic sections of the human brain. Microsc Res Tech 63:220-243.

Schumann CM, Amaral DG (2006) Stereological analysis of amygdala neuron number in autism. J Neurosci 26:7674-7679.

Selemon LD, Rajkowska G, Goldman-Rakic PS (1998) Elevated neuronal density in prefrontal area 46 in brains from schizophrenic patients: application of a three-dimensional, stereologic counting method. J Comp Neurol 392:402-412.

Stark AK, Uylings HB, Sanz-Arigita E, Pakkenberg B (2004) Glial cell loss in the anterior cingulate cortex, a subregion of the prefrontal cortex, in subjects with schizophrenia. Am J Psychiatry 161:882-888.

Südhof TC (2008) Neuroligins and neurexins link synaptic function to cognitive disease. Nature 455:903-911.

Sundaram SK, Kumar A, Makki MI, Behen ME, Chugani HT, Chugani DC (2008) Diffusion tensor imaging of frontal lobe in autism spectrum disorder. Cereb Cortex 18:2659-2665.

Thakkar KN, Polli FE, Joseph RM, Tuch DS, Hadjikhani N, Barton JJ, Manoach DS (2008) Response monitoring, repetitive behaviour and anterior cingulate abnormalities in autism spectrum disorders (ASD). Brain 131:2464-2478.

Van Essen DC (1997) A tension-based theory of morphogenesis and compact wiring in the central nervous system. Nature 385:313-318.

Vargas DL, Nascimbene C, Krishnan C, Zimmerman AW, Pardo CA (2005) Neuroglial activation and neuroinflammation in the brain of patients with autism. Ann Neurol 57:67-81. 
Von Bonin G (1950) Essay on the cerebral cortex. Springfield, IL: Thomas. von Economo C (2009) Cellular structure of the human cerebral cortex. Basel: Karger.

Walsh CA, Morrow EM, Rubenstein JL (2008) Autism and brain development. Cell 135:396-400.

Wang SS, Shultz JR, Burish MJ, Harrison KH, Hof PR, Towns LC, Wagers MW, Wyatt KD (2008) Functional trade-offs in white matter axonal scaling. J Neurosci 28:4047-4056.

Wang XJ, Tegnér J, Constantinidis C, Goldman-Rakic PS (2004) Division of labor among distinct subtypes of inhibitory neurons in a cortical microcircuit of working memory. Proc Natl Acad Sci U S A 101:1368-1373.

Weiss LA, Arking DE, Daly MJ, Chakravarti A (2009) A genome-wide linkage and association scan reveals novel loci for autism. Nature 461:802-808.

West MJ, Slomianka L, Gundersen HJ (1991) Unbiased stereological esti- mation of the total number of neurons in the subdivisions of the rat hippocampus using the optical fractionator. Anat Rec 231:482-497.

Wong KL, Murakami K, Routtenberg A (1989) Dietary cis-fatty acids that increase protein $\mathrm{F} 1$ phosphorylation enhance spatial memory. Brain Res 505:302-305.

Yakovlev PI, Lecours A-R (1967) The myelogenetic cycles of regional maturation of the brain. In: Regional development of the brain in early life (Minowski A, ed), pp 3-70. Oxford, Edinburgh: Blackwell.

Yip J, Soghomonian JJ, Blatt GJ (2007) Decreased GAD67 mRNA levels in cerebellar Purkinje cells in autism: pathophysiological implications. Acta Neuropathol 113:559-568.

Zikopoulos B, Barbas H (2006) Prefrontal projections to the thalamic reticular nucleus form a unique circuit for attentional mechanisms. J Neurosci 26:7348-7361.

Zikopoulos B, Barbas H (2007) Parallel driving and modulatory pathways link the prefrontal cortex and thalamus. PLoS One 2: e848. 